US-98-01

hep-th/9802043

\title{
Conformal Anomaly in 4D Gravity-Matter Theories Non-minimally Coupled with Dilaton
}

\author{
Shoichi ICHINOSE \& and Sergei D. ODINTSOV ${ }^{\dagger}$ ?
}

Department of Physics, Universuty of Shizuoka

Yada 52-1, Shizuoka 422-8526, JAPAN

†Tomsk Pedagogical University, 634041 Tomsk, RUSSIA

\begin{abstract}
The conformal anomaly for 4D gravity-matter theories, which are non-minimally coupled with the dilaton, is systematically studied. Special care is taken for: rescaling of fields, treatment of total derivatives, hermiticity of the system operator and choice of measure. Scalar, spinor and vector fields are taken as the matter quantum fields and their explicit conformal anomalies in the gravity-dilaton background are found. The cohomology analysis is done and some new conformal invariants and trivial terms, involving the dilaton, are obtained. The symmetry of the constant shift of the dilaton field plays an important role. The general structure of the conformal anomaly is examined. It is shown that the dilaton affects the conformal anomaly characteristically for each case: 1) [Scalar] The dilaton changes the conformal anomaly only by a new conformal invariant, $\left.I_{4} ; 2\right)$ [Spinor] The dilaton does not change the conformal anomaly; 3)[Vector] The dilaton changes the conformal anomaly

\footnotetext{
1 e-mail : ichinose@u-shizuoka-ken.ac.jp

2 e-mail : odintsov@tspi.tomsk.su
} 
by three new (generalized) conformal invariants, $I_{4}, I_{2}, I_{1}$. We present some new anomaly formulae which are useful for practical calculations. Finally, the anomaly induced action is calculated for the dilatonic Wess-Zumino model. We point out that the coefficient of the total derivative term in the conformal anomaly for the 2D scalar coupled to a dilaton is ambiguous. This resolves the disagreement between calculations in refs. [3, 4, 5, 6] and the result of Hawking-Bousso[2].

- PACS NO: 04.62.+v,11.25.Db,11.30.-j,14.80.-j

- Keywords: conformal anomaly, dilaton gravity, dilatonic Wess-Zumino model, anomaly formula

\section{Introduction}

Stimulated by string theory, the higher dimensional approach to the $4 \mathrm{D}$ theory becomes gradually important. Indeed it provides fruitful possibilities for theoretical analysis. Kaluza-Klein type dimensional reduction is the most popular one. However realizations of such reductions depend on the chosen higher dimension, the method of reduction, the choice of a symmetry ansatz, and so on. At present there seems to be no fixed rule to find the "right" one. We first note that, in low-energy effective actions, some characteristic fields, such as the dilaton and the anti-symmetric tensor commonly appear as remnants of the extra dimensions. Through the study of the dynamical effect of such fields, we can learn some common features of $4 \mathrm{D}$ effective theories that the higher-dimensional theories produce in the low energy limit.

We focus on the dilaton field for simplicity. Its effect on the Weyl anomaly is examined. We start from the Weyl invariant action in order to set up the problem clearly. The coupling of the dilaton is fixed by keeping the Weyl symmetry. The most characteristic aspect is its exponential form. Furthermore it couples to most fields just like the gravitational field itself. ( From 
the standpoint of the string theory or Kaluza-Klein theory, this is a natural aspect because the dilaton comes from a gravitational component in the compactified dimensions.) We treat the dilaton as the background field in the same way as the gravitational field. We take the standpoint that the quantum treatment of the dilaton, like the gravitational field, should be explained by the string theory.

It is interesting that the dilaton is expected to play a role of a coupling in string theory. Indeed, in the present treatment, the dilaton is invariant under all symmetry transformations and behaves just like a coupling constant except that it depends on the space coordinates. The dilaton transforms as a scalar under the general coordinate transformation. Under the Weyl transformation, it does not change

$$
\phi^{\prime}=\phi
$$

In this point the dilaton transforms differently from the scalar field except in the 2D space (see Appendix C), which is natural because of the dimensional difference between the dilaton and a scalar field.

As for the role of the dilaton, some interesting arguments have been presented recently. Even at the classical level, the dilaton can make the singularity behaviour weaker in the early universe cosmology [1]. In 2D or 4D black hole physics, its presence influences the Hawking radiation [2, 3, 0, 0, 6]. As for a recent development using the conformal anomaly, we note ref. [7] where a nonperturbative approach to the conformal field theory in higher dimensions is presented ( for early works in the study of higher dimensional conformal field theory, see 8). The conformal anomaly in 4D dilaton gravityvector has been recently found in [9] where the induced action is examined and by [10] where the general structure of the conformal anomaly is analysed.

The above is the general background to the present work. In the following we will concretely analyse the following points.

1. We take the spin=0,1/2,1 matter fields on dilatonic curved backgrounds and examine the conformal anomaly due to quantum effects. The dilaton's presence is a new point here.

2. We are interested in the general structure of the conformal anomaly. 
3. In the evaluation of the anomaly we pay attention to the following points: the arbitrariness of total derivatives or partial derivatives in the operators, rescaling of fields and the choice of measure.

4. We present some useful anomaly formulae and show how to use them.

5. We check the previous work, which is obtained as a special case : dilaton field $=$ const by using a different approach (heat-kernel regularization and Fujikawa method).

We begin with the explanation of the present formalism and the cohomology analysis in Sec.2. In Sec.3 the scalar theory is considered. This is the simplest case and is the best model in which the present analysis is explained. In Sec. 4 the vector theory is considered. We must take into account the gauge fixing which introduces some complication. It breaks the Weyl symmetry as well as the gauge symmetry. The final result is therefore highly non-trivial. In Sec.5 we treat the spinor theory. It turns out that the dilaton does not affect the spinor theory. Finally we discuss the anomaly induced effective action in Sec.6. As an example the dilatonic Wess-Zumino model is considered. We conclude in Sec.7. In appendix A and B, we derive useful anomaly formulae used in the text. We use heat-kernel regularization for the ultra-violet divergences and follow the Fujikawa method[11]. The results turn out to be the same as the counterterm formulae already obtained by other authors, where dimensional regularization was used and the (log-)divergent part of the effective action was calculated. In the present approach the anomaly term is definitely determined (no ambiguity of the total divergence) once the system operator (elliptic differential operator) and the regularization are fixed. In appendix C the Weyl transformations of various fields are listed. In appendix D, the list of "trivial terms" is presented. In appendix E we discuss the conformal anomaly for a two-dimensional dilaton coupled scalar.

\section{Formalism and Cohomology Analysis}

We consider the system of quantum matter (scalar,vector,spinor,etc.) fields $f(x)$, in the background metric $g_{\mu \nu}(x)$ and the background dilaton field $\phi(x)$, described by the action $S[f ; g, \phi]$. The (Wilsonian) effective action $\Gamma[g, \phi]$ is 
defined by

$$
\mathrm{e}^{\Gamma[g, \phi]}=\int \mathcal{D} f \exp \{S[f ; g, \phi]\}
$$

Under the Weyl transformation, the classical lagrangian is assumed to be invariant.

$$
\begin{array}{r}
\phi^{\prime}=\phi \quad, \quad g_{\mu \nu}{ }^{\prime}=\mathrm{e}^{2 \alpha(x)} g_{\mu \nu} \quad, \quad f^{\prime}=\mathrm{e}^{c \alpha(x)} f \\
S\left[f^{\prime} ; g^{\prime}, \phi^{\prime}=\phi\right]=S[f ; g, \phi]
\end{array},
$$

where $c$ is some constant (the scale dimension of the matter field). The effective action $\Gamma$ is generally changed due to the measure (quantum) effect which is the origin of the conformal anomaly [11]. The change is given by

$$
\left.\frac{\delta \Gamma}{\delta \alpha(x)}\right|_{\alpha=0}=2 g_{\mu \nu}(x) \frac{\delta \Gamma}{\delta g_{\mu \nu}(x)} \equiv 2 g_{\mu \nu}<T^{\mu \nu}>
$$

where $T^{\mu \nu}$ is the energy-momentum tensor. This quantity is the conformal (Weyl) anomaly and is evaluated as follows.

$$
\begin{gathered}
\mathrm{e}^{\Gamma\left[g^{\prime}, \phi^{\prime}=\phi\right]}=\int \mathcal{D} f^{\prime} \exp \left\{S\left[f^{\prime} ; g^{\prime}, \phi^{\prime}=\phi\right]\right\} \\
=\int \mathcal{D} f \operatorname{det}\left(\frac{\partial f^{\prime}}{\partial f}\right) \exp \{S[f ; g, \phi]\} .
\end{gathered}
$$

We can evaluate the Jacobian in the heat-kernel regularization.

$$
\begin{array}{r}
\ln \operatorname{det} \frac{\partial f^{\prime}}{\partial f}=\operatorname{Tr} \ln \left\{\mathrm{e}^{c \alpha(x)} \delta^{4}(x-y)\right\}=c \operatorname{Tr}\left\{\alpha(x) \delta^{4}(x-y)\right\}+O\left(\alpha^{2}\right) \\
=c \lim _{t \rightarrow+0} \operatorname{Tr}\left\{\alpha(x)<x\left|\mathrm{e}^{-t \vec{D}}\right| y>\right\}+O\left(\alpha^{2}\right) \\
g_{\mu \nu}<T^{\mu \nu}>=\left.\frac{c}{2} \operatorname{tr}\left\{<x\left|\mathrm{e}^{-t \vec{D}}\right| x>\right\}\right|_{t^{0}}
\end{array}
$$

By differentiating the equation (5) w.r.t.(with respect to) the background sources $g_{\mu \nu}$ and $\phi$, we can obtain various Ward-Takahashi identites which take into account the conformal anomaly. They correspond to 1-loop graphs of 2-point, 3-point, ... functions of corresponding currents. Taking $t^{0}$ part in (6) corresponds to considering the log-divergent part in the dimensional 
regularization. All these things and the fact eq.(6) correctly gives the all known anomalies (both chiral and Weyl) were confirmed in [12]. Our task therefore reduces to the evaluation of $\left.\operatorname{tr}\left\{\left\langle x\left|\mathrm{e}^{-t \vec{D}}\right| x\right\rangle\right\}\right|_{t^{0}}$.

It is well-known the anomaly can be fixed to some extent by the cohomology of the corresponding symmetry operators. It is powerful in that no explicit calculation is required (hence we need not to worry about the regularization ambiguity) and only some consistency relations due to the symmetries are essential. Especially for the chiral anomaly, the anomaly is fixed except one overall coefficient. Let us do the cohomology analysis for the present conformal anomaly.

Following Ref. 13 we consider the infinitesimal Weyl transformation in (3) $, \delta g_{\mu \nu}=2 \alpha(x) g_{\mu \nu}, \delta \phi=0$ and regard the infinitesimal parameter $\alpha(x)$ as an anti-commuting one. [3 Then we can introduce the coboundary operator $\Sigma$ which acts on the functional space of $g_{\mu \nu}$ and $\phi$.

$$
\Sigma=\int d^{4} x 2 \alpha(x) g_{\mu \nu}(x) \frac{\delta}{\delta g_{\mu \nu}(x)} \quad, \quad \Sigma^{2}=0
$$

which is a nilpotent operator. It acts on the effective action $\Gamma[g, \phi]$ as

$$
\Sigma \Gamma=\hbar \Delta+O\left(\hbar^{2}\right)
$$

where the right hand side is expanded w.r.t. the loop-number $(\hbar)$. In the anomaly, we usually consider only 1-loop part. $\Delta$, after removing "trivial terms" (see Appendix D), is defined as the anomaly in the cohomology. Because of the nilpotency property, $\Sigma^{2}=0$, the term $\Delta$ must satisfy the following consistency condition.

$$
\Sigma \Delta=0
$$

We impose the following conditions on the "basis" of the function space of $\Delta$.

1. $4 \mathrm{D}$ general coordinate invariance.

2. Physical dimension is 4 ( Number of derivatives is 4 ).

\footnotetext{
${ }^{3}$ Only from this paragraph to the end of this section, the Weyl transformation parameter $\alpha(x)$ is infinitesimal and anti-commuting.
} 
3. "Ghost" number is 1 . (This means we consider those terms which contain one $\alpha$ only.)

4. Symmetry of constant shift: $P(\phi) \rightarrow P(\phi)+$ const .

The last item 4 is the speciality of the dilaton field. (Compare with the scalar field considered in [13.) For the familiar case $P(\phi)=\phi$, the transformation is the constant shift of the dilaton. The actions involving the dilaton (see (13), (29) and (54) in the following text) are essentially invariant because the transformation changes the actions only by an overall constant factor, which can be absorbed by the constant rescaling of the matter fields: $f \rightarrow f \times$ const. This symmetry is very important because it forbids, as the anomaly terms, the appearance of the terms like $\sqrt{g}\left(P_{, \mu} P^{, \mu}\right)^{2} P^{2}, \sqrt{g}\left(P_{, \mu} P^{, \mu}\right)^{2} P^{4}, \sqrt{g} C_{\mu \nu \lambda \sigma} C^{\mu \nu \lambda \sigma} P^{2}, \cdots$

Let us see how the consistency condition (9) restricts $\Delta$. In Table 1 we list all the possible terms, $\Delta_{i}$, which satisfy the above four conditions and $\Sigma$-transformed ones for each, $\Sigma \Delta_{i}$.@ Before the consistency condition is imposed, the most general expression is given as

$$
\Delta=\sum_{i} c_{i} \Delta_{i}
$$

From the consistency condition we obtain the following relations between coefficients.

$$
\text { ii) } \begin{aligned}
\text { i) } c_{1}+c_{2}+c_{3}=0 \\
-2 c_{7}+4 c_{8}+2 c_{9}=0 \\
\text { iii) } 2 c_{14}-c_{15}=0 \\
\text { iv) } c_{16}+3 c_{19}=0 \quad, \quad 2 c_{17}+c_{18}=0
\end{aligned} \text {. }
$$

Other coefficients are arbitrary. 


\begin{tabular}{|c|c|c|}
\hline No & $\Delta_{i}$ & $\Sigma \Delta_{i}$ \\
\hline 1 & 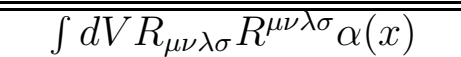 & 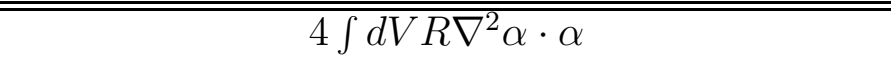 \\
\hline 2 & $\int d V R_{\mu \nu} R^{\mu \nu} \alpha(x)$ & $4 \int d V R \nabla^{2} \alpha \cdot \alpha$ \\
\hline 3 & $\int d V R^{2} \alpha(x)$ & $12 \int d V R \nabla^{2} \alpha \cdot \alpha$ \\
\hline 4 & $\int d V\left(\nabla^{2} R\right) \cdot \alpha(x)$ & 0 \\
\hline 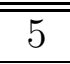 & $\int d V\left(P_{, \mu} P^{, \mu}\right)^{2} \alpha(x)$ & 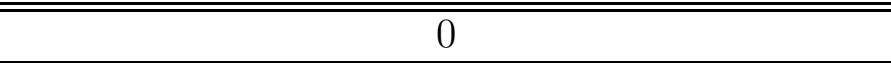 \\
\hline 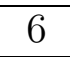 & $\int \overline{e d V R P_{, \mu} P^{, \mu} \alpha(x)}$ & 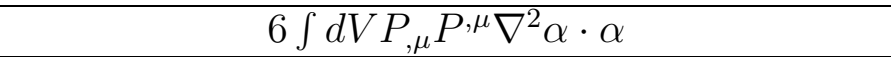 \\
\hline 7 & $\int d V R^{\mu \nu} P_{, \mu} P_{, \nu} \alpha(x)$ & $\int d V\left(P_{, \mu} P^{, \mu} \nabla^{2} \alpha-2 P^{, \mu \nu} P_{, \mu} \alpha_{, \nu}-2 \nabla^{2} P \cdot P^{, \mu} \alpha_{, \mu}\right) \alpha$ \\
\hline 8 & $\int d V\left(\nabla^{2} P\right)^{2} \cdot \alpha(x)$ & $4 \int d V \nabla^{2} P \cdot P^{, \mu} \alpha_{, \mu} \alpha$ \\
\hline 9 & $\int d V P_{, \mu \nu} P^{, \mu \nu} \alpha(x)$ & $\int d V\left(-4 P^{, \mu \nu} P_{, \mu} \alpha_{, \nu} \alpha+2 \nabla^{2} P \cdot P^{, \mu} \alpha_{, \mu} \alpha\right)$ \\
\hline 10 & $\int d V \nabla_{\mu}\left(\nabla^{2} P \cdot P^{, \mu}\right) \cdot \alpha(x)$ & 0 \\
\hline 11 & $\int d V \nabla_{\mu}\left(P^{, \mu \nu} P_{, \nu}\right) \cdot \alpha(x)$ & 0 \\
\hline 12 & $\int d V \nabla^{2}\left(P_{, \mu} P^{, \mu}\right) \cdot \alpha(x)$ & 0 \\
\hline 13 & $\int d V \nabla_{\mu} \nabla_{\nu}\left(P^{, \mu} P^{, \nu}\right) \cdot \alpha(x)$ & 0 \\
\hline 14 & $=\int d V P_{, \mu} P^{, \mu}\left(\nabla^{2} P\right) \cdot \alpha(x)$ & 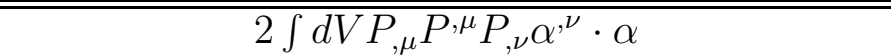 \\
\hline 15 & $\int d V P^{, \mu} P^{, \nu} P_{, \mu \nu} \alpha(x)$ & $-\int d V P_{, \mu} P^{, \mu} P_{, \nu} \alpha^{, \nu} \cdot \alpha$ \\
\hline 16 & $\int d V \nabla^{4} P \cdot \alpha(x)$ & $-2 \int d V P_{, \mu} \nabla^{2} \alpha \cdot \alpha^{, \mu}$ \\
\hline 17 & $\int d V R \nabla^{2} P \cdot \alpha(x)$ & $\int d V\left(6 \nabla^{2} P \cdot \nabla^{2} \alpha+2 R P_{, \mu} \alpha^{, \mu}\right) \cdot \alpha$ \\
\hline 18 & $\int d V R^{\mu \nu} P_{, \mu \nu} \cdot \alpha(x)$ & $\int d V\left(3 \nabla^{2} P \cdot \nabla^{2} \alpha+R P_{, \mu} \alpha^{, \mu}\right) \cdot \alpha$ \\
\hline 19 & $\int d V \nabla_{\mu}\left(R P^{, \mu}\right) \cdot \alpha(x)$ & $-6 \int d V P^{, \mu} \nabla^{2} \alpha \cdot \alpha_{, \mu}$ \\
\hline
\end{tabular}

Table 1 List of all possible terms $\Delta_{i}$ which satisfy the four conditions in Sec.2 and their $\Sigma$-transformed ones(see eq.(7)). $\int d V \equiv \int d^{4} x \sqrt{g}$.

Since we have 7 relations among 19 terms, $19-7=12$ terms remain as the possible Weyl anomaly terms appearing in the gravity-dilaton background system. Among the 12 terms, there are "trivial" terms which can be freely adjusted by introducing local conter-terms in the action. In order to find all trivial terms, we list, in Table 2, all the possible local counterterms $\gamma_{i}$ and the $\Sigma$-transformed ones for each, $\Sigma \gamma_{i}$. The symmetries which define the counterterms are the same as those for $\Delta$ except the condition 3. "Ghost" number is 0 . 


\begin{tabular}{|c|c|c|}
\hline No & $\gamma_{i}$ & $\Sigma \gamma_{i}$ \\
\hline \hline 1 & $\int d V R_{\mu \nu \lambda \sigma} R^{\mu \nu \lambda \sigma}$ & $4 \int d V \alpha \nabla^{2} R$ \\
\hline 2 & $\int d V R_{\mu \nu} R^{\mu \nu}$ & $4 \int d V \alpha \nabla^{2} R$ \\
\hline 3 & $\int d V R^{2}$ & $12 \int d V \alpha \nabla^{2} R$ \\
\hline 4 & $\int d V\left(\nabla^{2} R\right)$ & 0 \\
\hline \hline 5 & $\int d V\left(P_{, \mu} P^{, \mu}\right)^{2}$ & 0 \\
\hline \hline 6 & $\int d V R P_{, \mu} P^{, \mu}$ & $6 \int d V \alpha \nabla^{2}\left(P_{, \mu} P^{, \mu}\right)$ \\
\hline 7 & $\int d V R^{\mu \nu} P_{, \mu} P_{, \nu}$ & $\int d V \alpha\left\{\nabla^{2}\left(P_{, \mu} P^{, \mu}\right)+2 \nabla_{\mu} \nabla_{\nu}\left(P^{, \mu} P^{, \nu}\right)\right\}$ \\
\hline 8 & $\int d V\left(\nabla^{2} P\right)^{2}$ & $-4 \int d V \alpha \nabla_{\mu}\left(\nabla^{2} P \cdot P^{, \mu}\right)$ \\
\hline 9 & $\int d V P_{, \mu \nu} P^{, \mu \nu}$ & $2 \int d V \alpha \nabla_{\mu}\left(2 P^{, \mu \nu} P_{, \nu}-\nabla^{2} P \cdot P^{, \mu}\right)$ \\
\hline 10 & $\int d V \nabla_{\mu}\left(\nabla^{2} P^{\prime} \cdot P^{, \mu}\right)$ & 0 \\
\hline 11 & $\int d V \nabla_{\mu}\left(P^{, \mu \nu} P_{, \nu}\right)$ & 0 \\
\hline 12 & $\int d V \nabla^{2}\left(P_{, \mu} P^{, \mu}\right)$ & 0 \\
\hline 13 & $\int d V \nabla_{\mu} \nabla_{\nu}\left(P^{, \mu} P^{, \nu}\right)$ & 0 \\
\hline \hline 14 & $\int d V P_{, \mu} P^{, \mu}\left(\nabla^{2} P\right)$ & $-2 \int d V \alpha \nabla_{\nu}\left(P_{, \mu} P^{, \mu} P^{, \nu}\right)$ \\
\hline 15 & $\int d V P^{, \mu} P^{, \nu} P_{, \mu \nu}$ & $\int d V \alpha \nabla_{\nu}\left(P_{, \mu} P^{, \mu} P^{, \nu}\right)$ \\
\hline \hline 16 & $\int d V \nabla^{4} P$ & 0 \\
\hline 17 & $\int d V R \nabla^{2} P$ & $2 \int d V \alpha\left\{3 \nabla^{4} P_{-} \nabla^{\mu}\left(R P_{, \mu}\right)\right\}$ \\
\hline 18 & $\int d V R^{\mu \nu} P_{, \mu \nu}$ & $\int d V \alpha\left\{3 \nabla^{4} P-\nabla^{\mu}\left(R P_{, \mu}\right)\right\}$ \\
\hline 19 & $\int d V \nabla_{\mu}\left(R P^{, \mu}\right)$ & 0 \\
\hline
\end{tabular}

Table 2 List of all possible local counterterms $\gamma_{i}$ and their $\Sigma$-transformed ones. $\int d V \equiv \int d^{4} x \sqrt{g}$.

Therefore we conclude, from the cohomology analysis, the Weyl anomaly in the gravity-dilaton background system is composed of four invariants $\left(I_{0}, I_{4}, I_{2}, I_{1}\right)$, the Euler term $\sqrt{g} E$, seven trivial terms $\left(J_{0}, J_{2 a}, J_{2 b}, J_{2 c}, J_{2 d}, J_{3}, J_{1}\right)$. 4

$$
\frac{\delta}{\delta \alpha(x)} \Delta=b_{0} I_{0}+b_{E} \sqrt{g} E+c_{0} J_{0}+b_{4} I_{4}+b_{2} I_{2}+b_{1} I_{1}
$$

\footnotetext{
${ }^{4} I_{0} \sim \Delta_{1}-2 \Delta_{2}+(1 / 3) \Delta_{3}, \sqrt{g} E \sim \Delta_{1}-4 \Delta_{2}+\Delta_{3}, J_{0} \sim \Delta_{4}, I_{4} \sim \Delta_{5}, I_{2} \sim$ $-(1 / 6) \Delta_{6}+\Delta_{7}+(3 / 4) \Delta_{8}-(1 / 2) \Delta_{9}, I_{1} \sim-(1 / 2) \Delta_{17}+\Delta_{18}, J_{2 a} \sim \Delta_{12}, J_{2 b} \sim \Delta_{11}-$ $\Delta_{10}, J_{2 c} \sim \Delta_{13}, J_{2 d} \sim \Delta_{11}, J_{3} \sim \Delta_{14}+2 \Delta_{15}, J_{1} \sim 3 \Delta_{16}-\Delta_{19}$.
} 


$$
+c_{2 a} J_{2 a}+c_{2 b} J_{2 b}+c_{2 c} J_{2 c}+c_{2 d} J_{2 d}+c_{3} J_{3}+c_{1} J_{1} \quad .
$$

The definite expressions for the symbols $\left(I_{i}, E, J_{i}\right)$ appear in the following text and in Appendix D.

In the cohomology approach, there generally remains not a few undetermined coefficients of conformal anomaly terms, compared with the chiral anomaly. In order to fix them completely, the "full-fledged" dynamical calculation like the present approach is indispensable.

\section{Trace anomaly for 4D dilaton coupled scalar}

Let us start with the Lagrangian

$$
S_{1}[\varphi ; g, \phi]=\frac{1}{2} \int d^{4} x \sqrt{g} \mathrm{e}^{P(\phi)} \varphi\left(-\nabla^{2}+\xi R\right) \varphi \quad,
$$

where $\varphi, g_{\mu \nu}$ and $\phi$ are the scalar, graviton and dilaton fields, respectively. The form of $P(\phi)$ is often chosen to be const $\times \phi$, but we will keep a more general form. We treat $g_{\mu \nu}$ and $\phi$ as the background fields and the scalar matter field, $\varphi$, as the quantum field. At the value of $\xi=-\frac{1}{6}$, (13) is exactly (not ignoring total derivatives) conformally invariant. 向

$$
\phi^{\prime}=\phi \quad, \quad g_{\mu \nu}{ }^{\prime}=\mathrm{e}^{2 \alpha(x)} g_{\mu \nu} \quad, \quad \varphi^{\prime}=\mathrm{e}^{-\alpha(x)} \varphi .
$$

${ }^{5}$ We cannot take the following actions (compare it with the $2 \mathrm{D}$ case $[5]$ ):

$$
\begin{aligned}
S_{2}[\varphi ; g, \phi] & =\frac{1}{2} \int d^{4} x \sqrt{g} \mathrm{e}^{P(\phi)}\left(\nabla_{\mu} \varphi \cdot \nabla^{\mu} \varphi+\xi R \varphi^{2}\right) \\
S_{2}{ }^{\prime}[\varphi ; g, \phi] & =S_{2}[\varphi ; g, \phi]-\frac{1}{2} \int d^{4} x \sqrt{g} \nabla_{\mu}\left(\mathrm{e}^{P(\phi)} \varphi \nabla^{\mu} \varphi\right) \\
= & \frac{1}{2} \int d^{4} x \sqrt{g} \varphi \mathrm{e}^{P(\phi)}\left(-\nabla^{2}-P_{, \mu} \nabla^{\mu}+\xi R\right) \varphi .
\end{aligned}
$$

These two actions, in the $P(\phi)=$ const case, differ from (13) only by a total derivative. For the general case, however, they are not conformally invariant even for $\xi=-\frac{1}{6}$. The dilaton can select the coupling with the ordinary matter fields among some possibilities which differ only by the total derivative term (surface term) at the $P(\phi)=$ const limit. Note that the operator from $S_{2}{ }^{\prime}, \vec{D}_{2} \equiv \mathrm{e}^{P(\phi)}\left(-\nabla^{2}-P_{, \mu} \nabla^{\mu}+\xi R\right)$, is hermitian $\left(\vec{D}_{2}=\vec{D}_{2}^{\dagger}\right)$ for the natural definition: $\int d^{4} x \sqrt{g}\left(\vec{D}_{2} \varphi\right)^{\dagger} \varphi=\int d^{4} x \sqrt{g} \varphi^{\dagger} \vec{D}_{2}^{\dagger} \varphi$. 
In order to normalize the kinetic term of the scalar field, we rescale it as 回

$$
\Phi=\mathrm{e}^{\frac{1}{2} P(\phi)} \varphi \quad .
$$

Adding a total derivative term, $S_{1}$ changes to

$$
S=\frac{1}{2} \int d^{4} x \sqrt{g} \Phi\left\{-\nabla^{2}-\frac{1}{4} P_{, \mu} P^{, \mu}+\xi R\right\} \Phi=S_{1}-\frac{1}{4} \int d^{4} x \sqrt{g} \nabla_{\mu}\left(\Phi^{2} P^{, \mu}\right)
$$

We do the second rescaling in order to make the integration measure BRSTinvariant [14] with respect to (w.r.t.) 4D general coordinate symmetry.

$$
\tilde{\Phi}=\sqrt[4]{g} \Phi
$$

We can write $S$ as

$$
S=\frac{1}{2} \int d^{4} x \tilde{\Phi} \vec{D} \tilde{\Phi}, \vec{D} \equiv \sqrt[4]{g}\left(-\nabla^{2}-\overline{\mathcal{M}}\right) \frac{1}{\sqrt[4]{g}}, \overline{\mathcal{M}}=-\xi R+\frac{1}{4} P_{, \mu} P^{, \mu}
$$

We generally have the freedom of the choice of surface terms (like (17)) and of rescaling the variables ( like (16) and (18) ). We cannot keep $S_{1}$ as the action, because the corresponding operator $\vec{D}^{\prime}=\sqrt[4]{g}\left(-\nabla^{2}-\overline{\mathcal{M}}+\frac{1}{2} \nabla^{2} P+\right.$ $\left.P^{, \mu} \nabla_{\mu}\right) \frac{1}{\sqrt[4]{g}}$, is not hermitian due to the presence of the first derivative term. We can define the functional space $\mathcal{F}$, in terms of the eigen functions of $\vec{D}$, as :

$$
\vec{D} u_{n}=\lambda_{n} u_{n} \quad, \quad \mathcal{F}=\left\{\tilde{\Phi}=\sum_{n} c_{n} u_{n} \mid c_{n} \in \text { Complex }, n=0,1,2, \cdots\right\}
$$

The inner product between functions and the hermite conjugate of the operator $\vec{D}$ are defined as

$$
\begin{gathered}
<\tilde{\Phi}_{1} \mid \tilde{\Phi}_{2}>\equiv \int d^{4} x \tilde{\Phi}_{1}^{\dagger} \tilde{\Phi}_{2}=\int d^{4} x \sqrt{g} \Phi_{1}^{\dagger} \Phi_{2}=\int d^{4} x \sqrt{g} \mathrm{e}^{P(\phi)} \varphi_{1}^{\dagger} \varphi_{2} \\
<\vec{D} \tilde{\Phi}_{1}\left|\tilde{\Phi}_{2}>\equiv<\tilde{\Phi}_{1}\right| \vec{D}^{\dagger} \tilde{\Phi}_{2}>
\end{gathered}
$$

\footnotetext{
${ }^{6}$ A way to avoid the rescaling (16) is to take fully the dilaton coupling into the operator: $\vec{D}_{1}=\mathrm{e}^{P(\phi)}\left(-\nabla^{2}+\xi R\right)$. This is, however, not hermitian $\left(\vec{D}_{1} \neq\left(\vec{D}_{1}\right)^{\dagger}\right)$ for the natural definition: $\int d^{4} x \sqrt{g}\left(\vec{D}_{1} \varphi\right)^{\dagger} \varphi=\int d^{4} x \sqrt{g} \varphi^{\dagger}\left(\vec{D}_{1}\right)^{\dagger} \varphi$. It should be compared with $\vec{D}_{2}$ in the previous footnote.
} 
Under this definition, the hermiticity of the operator $\vec{D}$ in (19) holds true. Taking the eigen functions $\left\{u_{n}\right\}$ orthonormal, the integration measure is given by

$$
<u_{n} \mid u_{m}>=\int d^{4} x u_{n}^{\dagger} u_{m}=\delta_{n m} \quad, \quad \prod_{n} d c_{n} \equiv \mathcal{D} \tilde{\Phi}=\mathcal{D}\left(g^{1 / 4} \mathrm{e}^{\frac{1}{2} P(\phi)} \varphi\right)
$$

This rather standard procedure is important especially in the dilaton coupled system in order to precisely fix the integration measure [5]. We note that the requirement of the hermiticity of the system operator $\vec{D}$ plays an important role here.

In Appendix A, the anomaly formula for $\vec{D}=\sqrt[4]{g}\left(-\nabla^{2}-\mathcal{M}\right) \frac{1}{\sqrt[4]{g}}$ with arbitrary background scalar $\mathcal{M}$ is obtained in the present approach (the heat-kernel regularization and Fujikawa method):

$$
\begin{aligned}
& \text { Anomaly }=\frac{\sqrt{g}}{(4 \pi)^{2}} \operatorname{Tr}\left\{\frac{1}{6} \nabla^{2}\left(\mathcal{M}-\frac{1}{6} R\right)+\frac{1}{2}\left(\mathcal{M}-\frac{1}{6} R\right)^{2}\right. \\
& \left.+\frac{1}{180}\left(R_{\mu \nu \lambda \sigma} R^{\mu \nu \lambda \sigma}-R_{\mu \nu} R^{\mu \nu}+0 \times R^{2}-\nabla^{2} R\right) \cdot \mathbf{1}\right\},
\end{aligned}
$$

where "Tr" means "trace" over the field suffix $i$ : $\operatorname{Tr} \mathcal{M}=\sum_{i=1}^{N_{s}} \mathcal{M}_{i i}$ and $(\mathbf{1})_{i j}=\delta_{i j}$. Formula (23) ( and the vector version (41) appearing later ) is the same as the counterterm formula given in [15, 16, 17] where the dimensional regularization was used. Taking $N_{s}=1$ for the present case we find

$$
\begin{gathered}
\text { Anomaly }\left.\right|_{\xi}=\frac{\sqrt{g}}{(4 \pi)^{2}}\left\{\frac{1}{32}\left(P_{, \mu} P^{, \mu}\right)^{2}+\frac{1}{180}\left(R_{\mu \nu \lambda \sigma} R^{\mu \nu \lambda \sigma}-R_{\mu \nu} R^{\mu \nu}-\nabla^{2} R\right)\right. \\
\left.+\left(\xi+\frac{1}{6}\right)\left(-\frac{1}{6} \nabla^{2} R+\frac{1}{2}\left(\xi+\frac{1}{6}\right) R^{2}-\frac{1}{4} R P_{, \mu} P^{, \mu}\right)+\frac{1}{24} \nabla^{2}\left(P_{, \mu} P^{, \mu}\right)\right\}
\end{gathered}
$$

This result is consistent with [18] where dimensional regularization was used. The above result is not a conformal anomaly in the strict sense because $\vec{D}$ of (19) is not conformal invariant for the general value of $\xi$. At the classical conformal limit $\xi=-\frac{1}{6}$, the above result reduces to the "true" conformal anomaly:

$$
\begin{array}{r}
\text { Anomaly }\left.\right|_{\xi=-1 / 6}=\frac{\sqrt{g}}{(4 \pi)^{2}}\left\{\frac{1}{32}\left(P_{, \mu} P^{, \mu}\right)^{2}\right. \\
\left.+\frac{1}{360}\left(3 C_{\mu \nu \lambda \sigma} C^{\mu \nu \lambda \sigma}-E\right)-\frac{1}{180} \nabla^{2} R+\frac{1}{24} \nabla^{2}\left(P_{, \mu} P^{, \mu}\right)\right\}
\end{array}
$$


where $C_{\mu \nu \lambda \sigma}$ and $E$ are the conformal tensor(Appendix C) and the Euler term, respectively, which are defined by

$$
\begin{array}{r}
C_{\lambda \mu \nu \sigma}=R_{\lambda \mu \nu \sigma}+\frac{1}{2}\left(g_{\lambda \nu} R_{\mu \sigma}-g_{\lambda \sigma} R_{\mu \nu}+g_{\mu \sigma} R_{\lambda \nu}-g_{\mu \nu} R_{\lambda \sigma}\right) \\
+\frac{1}{6}\left(g_{\lambda \sigma} g_{\mu \nu}-g_{\lambda \nu} g_{\mu \sigma}\right) R \\
C_{\lambda \mu \nu \sigma} C^{\lambda \mu \nu \sigma}=-2 R_{\mu \nu} R^{\mu \nu}+R_{\mu \nu \lambda \sigma} R^{\mu \nu \lambda \sigma}+\frac{1}{3} R^{2} \\
E=\frac{1}{4} R_{\mu \nu \alpha \beta} R_{\lambda \sigma \gamma \delta} \epsilon^{\mu \nu \lambda \sigma} \epsilon^{\alpha \beta \gamma \delta}=R^{2}+R_{\mu \nu \alpha \beta} R^{\mu \nu \alpha \beta}-4 R_{\mu \nu} R^{\mu \nu}
\end{array}
$$

In (25) we notice two conformal invariants:

$$
I_{0} \equiv \sqrt{g} C_{\mu \nu \lambda \sigma} C^{\mu \nu \lambda \sigma}, I_{4} \equiv \sqrt{g}\left(P_{, \mu} P^{, \mu}\right)^{2},
$$

and two "trivial" terms (Appendix D) $J_{0}$ and $J_{2 a}$ :

$$
\begin{array}{r}
J_{0} \equiv \sqrt{g} \nabla^{2} R \quad, \quad K_{0} \equiv \sqrt{g} R^{2} \quad, \quad g_{\mu \nu} \frac{\delta}{\delta g_{\mu \nu}(x)} \int d^{4} x K_{0}=6 J_{0} \\
J_{2 a} \equiv \sqrt{g} \nabla^{2}\left(P_{, \mu} P^{, \mu}\right), K_{2 a} \equiv \sqrt{g} R P_{, \mu} P^{, \mu}, g_{\mu \nu} \frac{\delta}{\delta g_{\mu \nu}(x)} \int d^{4} x K_{2 a}=+3 J_{2 a}
\end{array}
$$

The numbers of lower suffixes in $I_{0}, I_{4}, J_{0}, K_{0}$, etc, show the power numbers of $P$ ( see Appendix D). We note that $I_{4}$ and $J_{2 a}$ are caused by the presence of the dilaton. Two terms $J_{0}$ and $J_{2 a}$ in (25) are arbitrarily adjusted by introducing finite counterterms, $K_{0}$ and $K_{2 a}$ respectively, in the original (local gravitational) action. This result says the conformal anomaly at $\xi=-\frac{1}{6}$ consists of two conformal invariants $\left(I_{0}, I_{4}\right)$, the Euler term $(\sqrt{g} E)$ and two trivial terms $\left(J_{0}, J_{2 a}\right)$. This confirms the general structure of the conformal anomaly discussed in [19, 20, 21, 10].

$2 \mathrm{D}$ case which is qualitatively the same is discussed in appendix E.

\section{Trace anomaly for $4 \mathrm{D}$ dilaton coupled vec- tor}

\subsection{Basic Formalism}

In this section we consider the dilaton coupled photon system. The action is given by 


$$
S_{V}[A ; g, \phi]=\int d^{4} x \sqrt{g}\left(-\frac{1}{4} \mathrm{e}^{P(\phi)} F_{\mu \nu} F^{\mu \nu}\right), F_{\mu \nu}=\nabla_{\mu} A_{\nu}-\nabla_{\nu} A_{\mu} .
$$

Besides the 4D general coordinate symmetry, this theory has the local Weyl symmetry:

$$
g_{\mu \nu}^{\prime}=\mathrm{e}^{2 \alpha(x)} g_{\mu \nu} \quad, \quad A_{\mu} \text { : fixed , } \phi: \text { fixed },
$$

and it has the Abelian local gauge symmetry:

$$
A_{\mu}^{\prime}=A_{\mu}+\nabla_{\mu} \Lambda(x) \quad, \quad g_{\mu \nu}: \text { fixed } \quad, \quad \phi: \text { fixed . }
$$

We take the following gauge-fixing term:

$$
S_{g f}[A ; g, \phi]=\int d^{4} x \sqrt{g}\left(-\frac{1}{2} \mathrm{e}^{P(\phi)}\left(\nabla_{\mu} A^{\mu}\right)^{2}\right)
$$

The corresponding ghost lagrangian is

$$
S_{g h}[\bar{c}, c ; g, \phi]=\int d^{4} x \sqrt{g} \mathrm{e}^{P(\phi)} \bar{c} \nabla_{\mu} \nabla^{\mu} c
$$

where $\bar{c}$ and $c$ are the anti-ghost and ghost fields respectively. They are hermitian scalar fields with Fermi statistics (Grassmannian variables). The total action $S[A, \bar{c}, c ; g, \phi]=S_{V}+S_{g f}+S_{g h}$ is invariant under the BRST symmetry

$$
\delta A_{\mu}=\xi \partial_{\mu} c \quad, \quad \delta \bar{c}=\xi \nabla^{\mu} A_{\mu} \quad, \quad \delta c=0 \quad, \quad \delta \phi=0 \quad, \quad \delta g_{\mu \nu}=0 \quad,
$$

where $\xi$ is the BRST parameter which is a global Grassmannian variable. $S_{g f}$ breaks the Weyl symmetry (30), besides the gauge symmetry (31). Therefore we must take close care to define the conformal anomaly in gauge theory. It is highly non-trivial whether the general structure suggested by Ref. 20, 21] holds true in this case.

We assign the following Weyl transformation to $\bar{c}$ and $c$ [22]:

$$
\bar{c}^{\prime}=\mathrm{e}^{-2 \alpha(x)} \bar{c} \quad, \quad c^{\prime}=c,
$$

which is chosen in the following way: i) the ghost action (33) is invariant under global Weyl transformation $(\alpha(x)$ is independent of $x)$; ii) $S_{g f}+S_{g h}$ 
is invariant, up to a "BRST-trivial" term, under infinitesimal local Weyl transformation.

After rescaling the vector and ghost fields ( for normalizing the kinetic term): $\mathrm{e}^{\frac{1}{2} P(\phi)} A_{\mu}=B_{\mu}, \mathrm{e}^{P(\phi)} \bar{c}=\bar{b}$, the actions can be expressed as

$$
\begin{gathered}
S_{V}+S_{g f}=\int d^{4} x\left[\frac{1}{2} \sqrt{g} B^{\mu}\left\{g_{\mu \nu} \nabla^{2}+\left(\mathcal{N}^{\lambda}\right)_{\mu \nu} \nabla_{\lambda}+\mathcal{M}_{\mu \nu}\right\} B^{\nu}+\text { total deri. }\right] \\
S_{g h}=\int d^{4} x \sqrt{g} \bar{b} \nabla^{2} c \\
\left(\mathcal{N}^{\lambda}\right)_{\mu \nu}=P_{, \mu} \delta_{\nu}^{\lambda}-P_{, \nu} \delta_{\mu}^{\lambda}, \quad \mathcal{M}_{\mu \nu}=R_{\mu \nu}-\frac{1}{4} g_{\mu \nu}\left(P_{, \lambda} P^{, \lambda}+2 \nabla^{2} P\right)
\end{gathered}
$$

Following Fujikawa 14, we take ( 4D general coordinate transformation symmetry) BRST-invariant measure by taking $\tilde{B}_{\mu}, \tilde{\bar{b}}$ and $\tilde{c}$ instead of $B_{\mu}, \bar{b}$ and $c$ respectively, as the path-integral variables ${ }^{7}$ :

$$
\begin{array}{r}
\tilde{B}_{\mu} \equiv g^{1 / 8} B_{\mu}\left(\tilde{B}^{\mu} \equiv g^{3 / 8} B^{\mu}\right) \quad \tilde{\bar{b}} \equiv \sqrt[4]{g} \bar{b} \quad, \tilde{c} \equiv \sqrt[4]{g} c \\
S_{V}+S_{g f}=\int d^{4} x\left[-\frac{1}{2} \tilde{B}^{\mu} \vec{D}_{\mu}^{\nu} \tilde{B}_{\nu}+\text { total deri. }\right], \\
\vec{D}_{\mu}{ }^{\nu}=-g^{1 / 8}\left\{\delta_{\mu}{ }^{\nu} \nabla^{2}+\left(\mathcal{N}^{\lambda}\right)_{\mu}{ }^{\nu} \nabla_{\lambda}+\mathcal{M}_{\mu}{ }^{\nu}\right\} g^{-1 / 8}, \\
S_{g h}=-\int d^{4} x \tilde{\bar{b}} \vec{d} \tilde{c} \quad, \quad \vec{d} \equiv-\sqrt[4]{g} \nabla^{2} \frac{1}{\sqrt[4]{g}}, \\
\exp \{\Gamma[g, \phi]\}=\int \mathcal{D} \tilde{B} \mathcal{D} \tilde{\bar{b}} \mathcal{D} \tilde{c} \exp (\tilde{S}[\tilde{B}, \tilde{\bar{b}}, \tilde{c} ; g, \phi]) \\
\tilde{S}[\tilde{B}, \tilde{\bar{b}}, \tilde{c} ; g, \phi]=S[A, \bar{c}, c ; g, \phi]
\end{array}
$$

where $\Gamma[g, \phi]$ is the (Wilsonian) effective action.

The Weyl transformation of (30) and that of the integration variables $\left(\tilde{B}_{\mu}^{\prime}=\mathrm{e}^{\alpha} \tilde{B}_{\mu}, \tilde{\bar{b}}^{\prime}=\tilde{\bar{b}}, \tilde{c}^{\prime}=\mathrm{e}^{2 \alpha} \tilde{c}\right)$ give us the Ward-Takahashi identity for the Weyl transformation:

$$
\begin{array}{r}
\exp \Gamma\left[g^{\prime}, \phi\right]=\int \mathcal{D} \tilde{B}^{\prime} \mathcal{D} \tilde{\bar{b}} \mathcal{D} \tilde{c}^{\prime} \exp \tilde{S}\left[\tilde{B}^{\prime}, \tilde{\bar{b}}, \tilde{c}^{\prime} ; g^{\prime}, \phi\right] \\
=\int \mathcal{D} \tilde{B} \cdot \operatorname{det} \frac{\partial \tilde{B}^{\prime}}{\partial \tilde{B}} \cdot \mathcal{D} \tilde{\bar{b}} \mathcal{D} \tilde{c} \cdot \operatorname{det} \frac{\partial \tilde{c}^{\prime}}{\partial \tilde{c}} \exp \tilde{S}\left[\tilde{B}^{\prime}, \tilde{\bar{b}}, \tilde{c}^{\prime} ; g^{\prime}, \phi\right] \\
=\int \mathcal{D} \tilde{B} \mathcal{D} \tilde{\bar{b}} \mathcal{D} \tilde{c} \cdot \operatorname{det}\left(\mathrm{e}^{\alpha} \delta_{\mu}{ }^{\nu} \delta^{4}(x-y)\right) \cdot\left\{\operatorname{det}\left(\mathrm{e}^{2 \alpha} \delta^{4}(x-y)\right)\right\}^{-1}
\end{array}
$$

7 The measure is also invariant under the BRST transformation w.r.t. the Abelian gauge symmetry: $\delta \tilde{B}_{\mu}=\xi \mathrm{e}^{P / 2} g^{-1 / 8}\left(\partial_{\mu} \tilde{c}-\frac{1}{4} g^{-1} \partial_{\mu} g \cdot \tilde{c}\right), \delta \tilde{\bar{b}}=\xi \mathrm{e}^{P / 2} g^{1 / 8}\left(\nabla^{\mu} \tilde{B}_{\mu}-\right.$ $\left.\frac{1}{8} g^{-1} g^{, \mu} \tilde{B}_{\mu}-\frac{1}{2} P^{, \mu} \tilde{B}_{\mu}\right), \delta \tilde{c}=0, \operatorname{det}\left\{\partial\left(\tilde{B}_{\mu}^{\prime}, \tilde{\bar{b}}^{\prime}, \tilde{c}^{\prime}\right) / \partial\left(\tilde{B}_{\nu}, \tilde{\bar{b}}, \tilde{c}\right)\right\}=1$. 


$$
\times \exp \left[\tilde{S}[\tilde{B}, \tilde{\bar{b}}, \tilde{c} ; g, \phi]+\delta \tilde{B}_{\mu} \frac{\delta \tilde{S}}{\delta \tilde{B}_{\mu}}+\delta \tilde{c} \frac{\delta \tilde{S}}{\delta \tilde{c}}+\delta g_{\mu \nu} \frac{\delta \tilde{S}}{\delta g_{\mu \nu}}\right]
$$

Considering the infinitesimal transformation $\left(\delta g_{\mu \nu}=2 \alpha g_{\mu \nu}, \delta \tilde{B}_{\mu}=\alpha \tilde{B}_{\mu}, \delta \tilde{c}=\right.$ $2 \alpha \tilde{c})$ in the above equation, and regularizing the space delta-functions $\delta^{4}(x-$ $y$ ) in terms of the heat-kernels, we obtain

$$
\begin{aligned}
\Gamma\left[g^{\prime}, \phi\right]-\Gamma[g, \phi]= & 2 \alpha g_{\mu \nu} \frac{\delta \Gamma}{\delta g_{\mu \nu}}=\alpha\left\langle\tilde{B}_{\mu} \frac{\delta \tilde{S}}{\delta \tilde{B}_{\mu}}\right\rangle+2 \alpha\left\langle\tilde{c} \frac{\delta \tilde{S}}{\delta \tilde{c}}\right\rangle+2 \alpha\left\langle g_{\mu \nu} \frac{\delta \tilde{S}}{\delta g_{\mu \nu}}\right\rangle \\
& +\operatorname{Tr} \ln \left\{\mathrm{e}^{\alpha}<x\left|\mathrm{e}^{-t \vec{D}_{\mu}{ }^{\nu}}\right| y>\right\}-\operatorname{Tr} \ln \left\{\mathrm{e}^{2 \alpha}<x\left|\mathrm{e}^{-t \vec{d}}\right| y>\right\},
\end{aligned}
$$

where $t$ is the regularization parameter: $t \rightarrow+0$. The part of variation w.r.t. $\tilde{S}$ gives the "naive" Ward-Takahashi identity, while the remaining two terms (terms with "Tr") give the deviation from it. (Compare this with the conformal gauge identities in gauge theories on curved space without the dilaton [17].) Therefore the last two terms can be regarded as the Weyl anomaly in this theory.

$$
\begin{gathered}
\left.T_{1} \equiv \frac{\partial}{\partial \alpha} \operatorname{Tr} \ln \left\{\mathrm{e}^{\alpha}<x\left|\mathrm{e}^{-t \vec{D}_{\mu}{ }^{\nu}}\right| y>\right\}\right|_{\alpha=0, t=0}, \\
T_{2} \equiv-\left.\frac{\partial}{\partial \alpha} \operatorname{Tr} \ln \left\{\mathrm{e}^{2 \alpha}<x\left|\mathrm{e}^{-t \vec{d}}\right| y>\right\}\right|_{\alpha=0, t=0},
\end{gathered}
$$

The anomaly formula for the differential operator $\vec{D}_{\mu}{ }^{\nu}=-g^{1 / 8}\left\{\delta_{\mu}{ }^{\nu} \nabla^{2}+\right.$ $\left.\left(\mathcal{N}^{\lambda}\right)_{\mu}{ }^{\nu} \nabla_{\lambda}+\mathcal{M}_{\mu}{ }^{\nu}\right\} g^{-1 / 8}$ with arbitrary general covariants of $\mathcal{N}^{\lambda}$ and $\mathcal{M}$ (taking the heat-kernel regularization and the Fujikawa method) is derived in Appendix B and is given as

$$
\begin{array}{r}
\text { Anomaly }=\frac{1}{(4 \pi)^{2}} \sqrt{g}\left\{\operatorname{Tr}\left[\frac{1}{6} D^{2} X+\frac{1}{2} X^{2}+\frac{1}{12} Y_{\alpha \beta} Y^{\alpha \beta}\right]\right. \\
\left.+4 \times \frac{1}{180}\left(R_{\mu \nu \lambda \sigma} R^{\mu \nu \lambda \sigma}-R_{\mu \nu} R^{\mu \nu}+0 \times R^{2}-\nabla^{2} R\right)\right\} \\
X_{\mu}{ }^{\nu}=\mathcal{M}_{\mu}{ }^{\nu}-\frac{1}{2}\left(\nabla_{\alpha} \mathcal{N}^{\alpha}\right)_{\mu}{ }^{\nu}-\frac{1}{4}\left(\mathcal{N}^{\alpha} \mathcal{N}_{\alpha}\right)_{\mu}{ }^{\nu}-\frac{1}{6} \delta_{\mu}{ }^{\nu} R \\
\left(Y_{\alpha \beta}\right)_{\mu}{ }^{\nu}=\left\{\frac{1}{2}\left(\nabla_{\alpha} \mathcal{N}_{\beta}\right)_{\mu}{ }^{\nu}+\frac{1}{4}\left(\mathcal{N}_{\alpha} \mathcal{N}_{\beta}\right)_{\mu}{ }^{\nu}-\alpha \leftrightarrow \beta\right\}+R_{\alpha \beta \mu}{ }^{\nu} \\
\left(D_{\alpha} X\right)_{\mu}{ }^{\nu}=\nabla_{\alpha} X_{\mu}{ }^{\nu}+\frac{1}{2}\left[\mathcal{N}_{\alpha}, X\right]_{\mu}{ }^{\nu},\left(D^{2} X\right)_{\mu}{ }^{\nu}=\nabla^{2} X_{\mu}{ }^{\nu}+\frac{1}{2}\left[\mathcal{N}^{\alpha}, D_{\alpha} X\right]_{\mu}{ }^{\nu} .
\end{array}
$$


Putting all explicit equations (36) into this formula, the vector contribution to the Weyl anomaly is finally given by

$$
\begin{aligned}
& T_{1}=\frac{1}{(4 \pi)^{2}} \sqrt{g}\left[\frac{1}{180}\left(-11 R_{\mu \nu \lambda \sigma} R^{\mu \nu \lambda \sigma}+86 R_{\mu \nu} R^{\mu \nu}-20 R^{2}+6 \nabla^{2} R\right)\right. \\
& +\left\{\frac{1}{16}\left(P_{, \mu} P^{, \mu}\right)^{2}+\frac{1}{12} \nabla^{2}\left(P_{, \mu} P^{, \mu}\right)+\frac{5}{12}\left(\nabla^{2} P\right)^{2}-\frac{1}{6} P_{, \mu \nu} P^{, \mu \nu}\right. \\
& \left.+\frac{2}{3} R^{\mu \nu} P_{, \mu} P_{, \nu}-\frac{1}{6} R P_{, \mu} P^{, \mu}\right\} \\
& \left.+\left\{-\frac{1}{3} \nabla^{4} P-\frac{1}{6}\left(P_{, \mu \nu}+\frac{1}{2} g_{\mu \nu} \nabla^{2} P\right) \cdot P^{, \mu} P^{, \nu}+\frac{1}{3}\left(R^{\mu \nu}-\frac{1}{2} g^{\mu \nu} R\right) P_{, \mu \nu}\right\}\right]
\end{aligned}
$$

Terms in the first big bracket $(\{\})$ show the even power part of $P(\phi)$, while those in the second show the odd power part. The ghost contribution to the Weyl anomaly is obtained from (23) with $\mathcal{M}=0$ as

$$
T_{2}=\frac{1}{(4 \pi)^{2}} \sqrt{g}\left[-2 \times \frac{1}{180}\left(-6 \nabla^{2} R+R_{\mu \nu \lambda \sigma} R^{\mu \nu \lambda \sigma}-R_{\mu \nu} R^{\mu \nu}+\frac{5}{2} R^{2}\right)\right]
$$

The factor -2 comes from the two fields of the ghosts $c$ and $\bar{c}$ and their Fermi statistics. There are no dilaton $(\mathrm{P})$-terms in the ghost part. $T_{1}+T_{2}$ gives the final result of the Weyl anomaly. It is given by the Euler term $(\sqrt{g} E)$, four Weyl invariants $\left(I_{4}, I_{2}, I_{1}, I_{0}\right)$ and five trivial terms $\left(J_{3}, J_{2 a}, J_{2 b}, J_{1}, J_{0}\right)$ which are defined in Appendix D:

$$
\begin{array}{r}
\text { Anomaly }=\frac{1}{(4 \pi)^{2}}\left\{\frac{1}{180}\left(-31 \sqrt{g} E+18 I_{0}+18 J_{0}\right)\right. \\
\left.+\frac{1}{16} I_{4}+I_{2}+\frac{1}{3} I_{1}-\frac{1}{3} \sqrt{g} \nabla^{4} P-\frac{1}{12} J_{3}+\frac{1}{12} J_{2 a}+\frac{1}{3} J_{2 b}\right\}
\end{array}
$$

except the term of $(-1 / 3) \sqrt{g} \nabla^{4} P$. In the next subsection, we show this term, combined with the contribution from the measure ambiguity, reduces to $(-2 / 15) J_{1}$. This result again confirms the general structure of the conformal anomaly analysed in [19, 20, 21, 10]. A slightly generalized aspect of the above result is that the new terms, $I_{2}$ and $I_{1}$, are conformally invariant not exactly but only up to total derivative terms. See appendix D for details. The conformal anomaly for the ordinary photon-graviton theory (without the dilaton) is correctly reproduced for $P(\phi)=$ const [23, 24, 25]. 


\subsection{Close treatment of Faddeev-Popov procedure and the integration variables}

In the previous section, the final result has the term $\sqrt{g} \nabla^{4} P$-term which is, by itself, not allowed by the cohomology analysis. We miss some contribution. First we notice one unsatisfactory treatment of the Faddeev-Popov procedure in the present ghost action (33). It originates from the "insertion" of the following identity in the path-integral expression.

$$
\begin{array}{r}
1=\int \Delta_{F} \delta\left(\sqrt[4]{g} \mathrm{e}^{\frac{1}{2} P(\phi)} \nabla_{\mu} A_{\Lambda}^{\mu}-K\right) \mathcal{D} \Lambda, \\
\Delta_{F}=\operatorname{det}\left(\sqrt[4]{g} \mathrm{e}^{\frac{1}{2} P} \nabla_{\mu} \nabla^{\mu}\right)=\operatorname{det}\left(\frac{1}{\sqrt[4]{g}} \mathrm{e}^{-\frac{1}{2} P}\right) \operatorname{det}\left(\sqrt{g} \mathrm{e}^{P} \nabla_{\mu} \nabla^{\mu}\right),
\end{array}
$$

where $K$ is a fixed constant and $\Lambda(x)$ is the abelian local gauge parameter (31). @ $\Delta_{F}$ is the "rigorous" F-P determinant whereas $\tilde{\Delta}_{F} \equiv \operatorname{det}\left(\sqrt{g} \mathrm{e}^{P} \nabla_{\mu} \nabla^{\mu}\right)$ is the one we have taken into account by (33). We must take care of the difference factor $\operatorname{det}\left(\frac{1}{\sqrt[4]{g}} \mathrm{e}^{-\frac{1}{2} P}\right)$, especially its $P$-dependent factor $\Delta_{P} \equiv \operatorname{det}\left(\mathrm{e}^{-\frac{1}{2} P}\right)$ for the present interest. (We notice the similar situation occurred in the gauge parameter (in)dependence problem of the $\nabla^{2} R$ anomaly-term in 4D vector model in the background metric (no dilaton) [34. The careful treatment of such a factor as above solves the problem. We see, from the gaugefixing term (32), $\mathrm{e}^{\frac{1}{2} P}$ corresponds to the "gauge parameter". ) Furthermore we notice such factors as $\Delta_{P}$, depending on the choice of the path-integral variables, can appear. For example, in eq.(36), such factors, depending on the choice of $\left(A_{\mu}, \bar{c}\right)$ or $\left(B_{\mu}, \bar{b}\right)$, indeed appear. (This factor is important when the overall factor of the path-integral get involved with the problem. In the case of Ref. 34], the overall factor depends on the gauge parameter. It affects the Weyl anomaly. In the present case, it depends on the dilaton field $P(\phi)$.) 8 As far as the dilaton is treated as the bacground field, we have arbitrariness of such a factor. Therefore we may assume there exists a factor $\Delta_{P}(\alpha) \equiv \operatorname{det}\left(\mathrm{e}^{\alpha P}\right)$, with some number $\alpha$, which multiplies the path-integral we are considering. Let us evaluate its contribution to the Weyl anomaly.

We consider the case $|P| \ll 1$ which is the region where the perturbative definition can be safely applied. Then the determinant factor can be

\footnotetext{
${ }^{8}$ In the string theory, the dilaton factor $\mathrm{e}^{P(\phi)}$ is regarded as a coupling. In the present problem the dilaton term behaves like the gauge parameter.
} 
evaluated, in the heat-kernel regularization, as

$$
\begin{aligned}
\ln \Delta_{P}(\alpha)=\ln \operatorname{det}\left\{\mathrm{e}^{\alpha P(\phi(x))} \delta^{(4)}(x-y)\right\} & =\alpha \lim _{t \rightarrow+0} \operatorname{Tr}\left\{P(\phi(x))<x\left|\mathrm{e}^{-t \vec{d}}\right| y>\right\} \\
& =\alpha \lim _{t \rightarrow+0} \frac{1}{t} \operatorname{Tr}<x\left|\mathrm{e}^{-t(\vec{d}-P)}\right| y>\left.\right|_{P^{1}},
\end{aligned}
$$

where $\vec{d}$ is given in (37). This trick of exponentiating the factor $P$ was used in [26]. Let us evaluate the heat-kernel

$$
\begin{gathered}
G^{P}(x, y ; t) \equiv \operatorname{Tr}<x\left|\mathrm{e}^{-t(\vec{d}-P)}\right| y>\quad \\
\left(\frac{\partial}{\partial t}+\vec{d}-P\right) G^{P}(x, y ; t)=0 .
\end{gathered}
$$

at the order of $O\left(P^{1}\right)$ w.r.t. the dilaton field $P$, and of $O(t)$ w.r.t. the proper time $t$. As for the background metric, it is sufficient to consider up to the first order of $O(h)$ w.r.t. the weak-field $h_{\mu \nu}\left(g_{\mu \nu}=\delta_{\mu \nu}+h_{\mu \nu},\left|h_{\mu \nu}\right| \ll 1\right)$. Their trace w.r.t. the space coordinates $(x=y)$ is given by $G_{1}(x, x ; t)$ and $G_{2}(x, x ; t)$ appearing in eq.(24) and eq.(25) of [12] respectively. Taking $n=4, M=-\frac{1}{4} \partial^{2} h+P+O\left(h^{2}\right), W_{\mu \nu}=-h_{\mu \nu}+O\left(h^{2}\right), N_{\lambda}=-\partial_{\mu} h_{\lambda \mu}+O\left(h^{2}\right)$ in the reference we obtain the following things. $\left(G^{P}(x, x ; t)=G_{0}(x, x ; t)+\right.$ $\left.G_{1}(x, x ; t)+G_{2}(x, x ; t)+\cdots.\right)$

$$
\text { (i) } G_{1}(x, x ; t)
$$

From (24) of [12], we obtain

$$
\begin{array}{r}
\frac{1}{(4 \pi)^{4} t} \int d^{4} w \int_{0}^{1} d r \frac{1}{\{(1-r) r\}^{2}} e^{-\frac{w^{2}}{4(1-r)}} \\
\times \frac{1}{4 !} \partial_{\alpha} \partial_{\beta} \partial_{\gamma} \partial_{\delta} P \cdot(\sqrt{t})^{4} w^{\alpha} w^{\beta} w^{\gamma} w^{\delta} e^{-\frac{w^{2}}{4 r}}=\frac{1}{(4 \pi)^{2}} \frac{t}{60} \partial^{4} P .
\end{array}
$$

(ii) $G_{2}(x, x ; t)$

From (25) of [12], the relevent part of $\partial^{3} h \cdot \partial P$ is given by

$$
\begin{array}{r}
\frac{1}{(4 \pi)^{6}} \int d^{4} v \int d^{4} u \int_{0}^{1} d k \int_{0}^{k} d l \frac{1}{\{(1-k)(k-l) l\}^{2}} e^{-\frac{v^{2}}{4(1-k)}} \\
\times\left[\partial _ { \omega } P \sqrt { t } v ^ { \omega } \mathrm { e } ^ { - \frac { ( v - u ) ^ { 2 } } { 4 ( k - l ) } } \left\{\frac{1}{t} \frac{(\sqrt{t})^{3}}{3 !} \partial_{\alpha} \partial_{\beta} \partial_{\gamma}\left(-h_{\mu \nu}\right) \cdot u^{\alpha} u^{\beta} u^{\gamma} \frac{\partial}{\partial u^{\mu}} \frac{\partial}{\partial u^{\nu}}\right.\right. \\
\left.+\frac{1}{\sqrt{t}} \frac{(\sqrt{t})^{2}}{2 !} \partial_{\alpha} \partial_{\beta}\left(-\partial_{\lambda} h_{\mu \lambda}\right) \cdot u^{\alpha} u^{\beta} \frac{\partial}{\partial u^{\mu}}+\sqrt{t} \partial_{\alpha}\left(-\frac{1}{4} \partial^{2} h\right) \cdot u^{\alpha}\right\} \mathrm{e}^{-\frac{u^{2}}{4 l}}
\end{array}
$$




$$
\begin{array}{r}
+\left\{\frac{1}{t} \frac{(\sqrt{t})^{3}}{3 !} \partial_{\alpha} \partial_{\beta} \partial_{\gamma}\left(-h_{\mu \nu}\right) \cdot v^{\alpha} v^{\beta} v^{\gamma} \frac{\partial}{\partial v^{\mu}} \frac{\partial}{\partial v^{\nu}}+\frac{1}{\sqrt{t}} \frac{(\sqrt{t})^{2}}{2 !} \partial_{\alpha} \partial_{\beta}\left(-\partial_{\lambda} h_{\mu \lambda}\right) \cdot v^{\alpha} v^{\beta} \frac{\partial}{\partial v^{\mu}}\right. \\
\left.\left.+\sqrt{t} \partial_{\alpha}\left(-\frac{1}{4} \partial^{2} h\right) \cdot v^{\alpha}\right\} \mathrm{e}^{-\frac{(v-u)^{2}}{4(k-l)}} \times \partial_{\omega} P \cdot \sqrt{t} u^{\omega} \mathrm{e}^{-\frac{u^{2}}{4 l}}\right] \\
=\frac{t}{(4 \pi)^{2}}\left[-\frac{1}{40} \partial_{\mu} \partial^{2} h_{\nu \nu} \cdot \partial_{\mu} P+\frac{1}{30} \partial_{\mu} \partial_{\nu} \partial_{\lambda} h_{\nu \lambda} \cdot \partial_{\mu} P+\text { other terns }\right]
\end{array}
$$

Making use of the weak-field expansion expressions

$$
\begin{gathered}
\nabla^{4} P=\partial^{4} P+\frac{1}{2} \partial_{\mu} \partial^{2} h \cdot \partial_{\mu} P-\partial^{2} \partial_{\mu} h_{\mu \nu} \cdot \partial_{\nu} P+O\left(h^{2}\right) \\
\nabla_{\lambda} R \cdot P^{, \lambda}=\partial_{\mu} \partial^{2} h \cdot \partial_{\mu} P-\partial_{\mu} \partial_{\nu} \partial_{\lambda} h_{\nu \lambda} \cdot \partial_{\mu} P+O\left(h^{2}\right)
\end{gathered}
$$

we demand the requirement of the cohomolgy analysis, that is, equating the following quantity

$$
\begin{array}{r}
\frac{1}{(4 \pi)^{2}}\left(-\frac{1}{3}\right) \nabla^{4} P+\ln \Delta_{P}(\alpha)=\frac{1}{(4 \pi)^{2}}\left(-\frac{1}{3}\right) \nabla^{4} P+\left.\alpha \lim _{t \rightarrow+0} \frac{1}{t} \operatorname{Tr} G^{P}(x, y ; t)\right|_{P^{1}} \\
=\frac{1}{(4 \pi)^{2}}\left(\left(\frac{\alpha}{60}-\frac{1}{3}\right) \partial^{4} P-\left(\frac{\alpha}{40}+\frac{1}{6}\right) \partial_{\mu} \partial^{2} h \cdot \partial_{\mu} P+\frac{\alpha}{30} \partial_{\mu} \partial_{\nu} \partial_{\lambda} h_{\nu \lambda} \cdot \partial_{\mu} P\right. \\
\text { +other terms }),(51)
\end{array}
$$

with the trivial term

$$
\begin{array}{r}
\beta \times J_{1}=\beta \sqrt{g}\left(3 \nabla^{4} P-\nabla^{\lambda}\left(R P_{, \lambda}\right)\right) \\
=\beta\left\{3 \partial^{4} P+\frac{1}{2} \partial_{\mu} \partial^{2} h \cdot P_{, \mu}+\partial_{\mu} \partial_{\nu} \partial_{\lambda} h_{\nu \lambda} \cdot P_{, \mu}\right\}+\text { other terms }
\end{array}
$$

we find

$$
\alpha=-4 \quad, \quad \beta=\frac{1}{(4 \pi)^{2}} \times\left(-\frac{2}{15}\right)
$$

The consistency with the cohomology determines the dilaton-depending overall factor of the path-integral. 


\section{Trace anomaly for 4D dilaton coupled spinor}

The dilaton can be introduced into the (Dirac) spinor-gravity system keeping the Weyl invariance as follows:

$$
\begin{array}{r}
S[\psi, \bar{\psi} ; g, \phi]=\frac{1}{2} \int d^{4} x \sqrt{g}^{P(\phi)}\{\bar{\psi} i \overleftrightarrow{\nabla} \psi\}, \bar{\psi} \overleftrightarrow{\nabla} \psi=\bar{\psi}\left(\gamma^{\mu} \nabla_{\mu} \psi\right)-\left(\bar{\psi} \overleftarrow{\nabla}_{\mu} \gamma^{\mu}\right) \psi \\
\not=\gamma^{\mu} \nabla_{\mu} \quad, \quad \nabla_{\mu} \psi=\left(\partial_{\mu}+\omega_{\mu}\right) \psi \quad, \quad \bar{\psi} \overleftarrow{\nabla}_{\mu}=\bar{\psi}\left(\overleftarrow{\partial}_{\mu}-\omega_{\mu}\right) \\
\omega_{\mu b}{ }^{a}=\left(\Gamma_{\mu \nu}^{\lambda} e_{\lambda}{ }^{a}-\partial_{\mu} e_{\nu}{ }^{a}\right) e^{\nu}{ }_{b} \quad, \quad \omega_{\mu}=\frac{1}{2} \sigma^{a b} \omega_{\mu a b}
\end{array}
$$

It is invariant under the Weyl transformation:

$$
\bar{\psi}^{\prime}=\mathrm{e}^{-\frac{3}{2} \alpha} \bar{\psi} \quad, \quad \psi^{\prime}=\mathrm{e}^{-\frac{3}{2} \alpha} \psi \quad, \quad \mathrm{e}_{\mu}^{a^{\prime}}=\mathrm{e}^{\alpha} \mathrm{e}_{\mu}^{a} \quad, \quad \phi^{\prime}=\phi \quad .
$$

We can absorb all $P(\phi)$ dependence by the rescaling of fields:

$$
\begin{array}{r}
\mathrm{e}^{\frac{1}{2} P(\phi)} \psi=\chi \quad, \quad \mathrm{e}^{\frac{1}{2} P(\phi)} \bar{\psi}=\bar{\chi} \\
S=\frac{1}{2} \int d^{4} x \sqrt{g} \bar{\chi} i \overleftrightarrow{\nabla} \chi=\frac{1}{2} \int d^{4} x \tilde{\bar{\chi}} \sqrt[4]{g} i \overleftrightarrow{\nabla} \frac{1}{\sqrt[4]{g}} \tilde{\chi}
\end{array}
$$

where $\tilde{\bar{\chi}}=\sqrt[4]{g} \bar{\chi}$ and $\tilde{\chi}=\sqrt[4]{g} \chi$. Therefore the dilaton does not change the conformal anomaly in the spinor-gravity theory as far as we take the appropriate measure: $\mathcal{D} \tilde{\bar{\chi}} \mathcal{D} \tilde{\chi}=\mathcal{D}\left(\sqrt[4]{g} \mathrm{e}^{P / 2} \psi\right) \mathcal{D}\left(\sqrt[4]{g} \mathrm{e}^{P / 2} \bar{\psi}\right)$.

\section{Induced Action for Dilatonic Wess-Zumino model}

In the present section we apply the previous results to the dilatonic WessZumino (WZ) model and discuss the conformal anomaly and the anomaly induced action. The model may be constructed following the book by Wess and Bagger 27. We suppose that the model is coupled with the external gravitational multiplet and the external dilaton (chiral) multiplet $\Psi$. Then using the notation of above book we may write the conformally invariant action:

$$
S=\int d^{2} \Theta 2 \mathcal{E}\left[-\frac{1}{16}(\overline{\mathcal{D}} \overline{\mathcal{D}}+8 R)\left\{\Phi^{\dagger}\left(\mathrm{e}^{P(\Psi)} \Phi\right)+\mathrm{e}^{P\left(\Psi^{\dagger}\right)} \Phi^{\dagger} \Phi\right\}\right]
$$


where $\Phi=A+\sqrt{2} \Theta \chi+\ldots$ and $\Psi=\phi+\ldots$. Choosing only the bosonic background for $\Psi$ and eliminating the auxiliary fields leads to the following component form of WZ action.

$$
L=e \mathrm{e}^{P(\phi)}\left\{-A \nabla^{2} A^{*}-\frac{1}{6} R A A^{*}-\frac{i}{2}\left(\chi \sigma^{\mu} \mathcal{D}_{\mu} \bar{\chi}+\bar{\chi} \sigma^{\mu} \mathcal{D}_{\mu} \chi\right)\right\},
$$

where we take the choice $\phi^{*}=\phi$ for the dilaton. $A$ is the complex scalar and $\chi$ is the Majorana spinor.

The conformal anomaly for the system may be found as follows

$$
\begin{gathered}
T=\frac{1}{(4 \pi)^{2}}\left\{b I_{0}+b^{\prime} \sqrt{g} E+b^{\prime \prime} J_{0}+a I_{4}+a^{\prime} J_{2 a}\right\}, \\
a=\frac{1 \times 2}{32}=\frac{1}{16}, a^{\prime}=\frac{1 \times 2}{24}=\frac{1}{12}, b=\frac{1}{120}\left(2+6 \times \frac{1}{2}\right)=\frac{1}{24}, \\
b^{\prime}=-\frac{1}{360}\left(2+11 \times \frac{1}{2}\right)=-\frac{1}{48}, b^{\prime \prime}=-\frac{1}{180}\left(2+6 \times \frac{1}{2}\right)=-\frac{1}{36} .
\end{gathered}
$$

$b^{\prime \prime}$ and $a^{\prime}$ are ambiguous as they may be changed by some finite counterterms in the gravitational action.

The nonlocal effective action may be found by integrating the Weyltransformed anomaly equation: f

$$
T\left(\mathrm{e}^{2 \sigma} g_{\mu \nu}\right)=\frac{1}{2} \frac{\delta}{\delta \sigma} W\left(\mathrm{e}^{2 \sigma} g_{\mu \nu}\right) .
$$

The final expression for the anomaly induced effective action $W$ is given as

$$
\begin{gathered}
W\left(g_{\mu \nu}\right)=\frac{1}{(4 \pi)^{2}} \int d^{4} x d^{4} y\left\{b I_{0}+a I_{4}+\frac{b^{\prime}}{2}\left(\sqrt{g} E+\frac{2}{3} J_{0}\right)\right\}_{x} G(x, y)\left\{\frac{1}{4}\left(\sqrt{g} E+\frac{2}{3} J_{0}\right)\right\}_{y} \\
+\frac{1}{(4 \pi)^{2}} \int d^{4} x\left\{\frac{1}{12}\left(b^{\prime \prime}-\frac{2}{3} b^{\prime}\right) K_{0}+\frac{a^{\prime}}{6} K_{2 a}\right\}
\end{gathered}
$$

where $W$ is in a covariant but non-local form via

$$
(\sqrt{g} \Delta)_{x} G(x, y)=\delta^{4}(x-y), \Delta=\left(\nabla^{2}\right)^{2}-2 R^{\mu \nu} \nabla_{\mu} \nabla_{\nu}+\frac{2}{3} R \nabla^{2}-\frac{1}{3}\left(\nabla^{\mu} R\right) \nabla_{\mu} .
$$

Here $\Delta$ is the conformally invariant 4-th order differential operator (Appendix D). For the case $a=a^{\prime}=0$, the result (61) coincides with Ref.[28]. Note that it is the effective action (61) that may serve as a starting point for constructing IR sector of the dilatonic gravity in the analogy with the purely gravity case of [31, 32].

\footnotetext{
${ }^{9}$ See [28, 29, 30] for the purely gravitational case and [18] for the dilaton-gravitational case.
} 
From another point using eq. 61) one can study the backreaction problem for the dilatonic WZ model. Here new possibilities are expected to appear in constructing the inflationary universe induced by the model. We hope to return to this question in more detail in future.

\section{Conclusion}

We have shown how the dilaton affects the conformal anomaly in various gravity-matter theories. The dilaton has the same status as the gravitational field. We summarize the results of this paper by writing the anomaly $T_{S}, T_{V}$ and $T_{F}$ for the scalar, the vector and the Dirac fermion respectively:

$$
\begin{gathered}
T_{S}=\frac{1}{(4 \pi)^{2}}\left\{\frac{1}{360}\left(-\sqrt{g} E+3 I_{0}\right)+\frac{1}{32} I_{4}+\text { Trivial Terms }\right\} \\
T_{V}=\frac{1}{(4 \pi)^{2}}\left\{\frac{1}{180}\left(-31 \sqrt{g} E+18 I_{0}\right)+\frac{1}{16} I_{4}+I_{2}+\frac{1}{3} I_{1}+\text { Trivial Terms }\right\} \\
T_{F}=\frac{1}{(4 \pi)^{2}}\left\{\frac{1}{180}\left(-\frac{11}{2} \sqrt{g} E+9 I_{0}\right)+\text { Trivial Terms }\right\} .
\end{gathered}
$$

For the system with $N_{S}$ scalars, $N_{F}$ Dirac fermions and $N_{V}$ vectors, the conformal anomaly is given by

$$
\begin{gathered}
\text { Anomaly }=\frac{1}{(4 \pi)^{2}}\left\{b_{E} \sqrt{g} E+b_{0} I_{0}+b_{4} I_{4}+b_{2} I_{2}+b_{1} I_{1}+\text { Trivial Terms }\right\} \\
b_{E}=\frac{1}{360}\left(-N_{S}-62 N_{V}-11 N_{F}\right)<0, b_{0}=\frac{1}{120}\left(N_{S}+12 N_{V}+6 N_{F}\right)>0, \\
b_{4}=\frac{1}{32}\left(N_{S}+2 N_{V}+0 \times N_{F}\right)>0, b_{2}=0 \times N_{S}+N_{V}+0 \times N_{F}>0 \\
b_{1}=0 \times N_{S}+\frac{1}{3} N_{V}+0 \times N_{F}>0 .
\end{gathered}
$$

This is the dilaton generalization of a result in Ref. 20. "Trivial terms" depend on the choice of regularization, measure and finite counterterms [5]. Their meaning is not very clear ( at least, in the perturbative approach). T0 On the other hand, the coefficients $b$ 's are clearly obtained and depend only on the content of the matter fields. They remind us of the critical indices in condensed matter physics.

In Sec.5 we have presented the anomaly induced action for the dilatonic Wess-Zumino model by integrating the conformal anomaly equation w.r.t. the Weyl mode. It is known that some anomaly equations like those of QED

\footnotetext{
10 The gauge (in)dependence of the "trivial terms" was analysed in Ref. 33. and the gauge independence was explicitly shown in Ref. 34.
} 
or $\lambda \phi^{4}$ theory on the curved space (with account of matter interaction) can not be integrated because of the presence of $R^{2}$-term in the anomaly [28]. Recently, in Ref.[9] it is noted that the anomaly for the free photon on the dilatonic curved space (44), cannot be integrated because of the presence of the new type (generalized) conformal invariants $I_{2}, I_{1 a}$ and $I_{1 b}$. In other words, a covariant anomaly induced effective action cannot be constructed for a dilaton coupled vector. It indicates some speciality of the gauge theory in comparison with the scalar and fermion fields. It could be possible to construct only non-covariant anomaly-induced effective action in the dilatoncoupled vector case. Probably, such an action still could lead to a correct conformal anomaly like in the case with Chern-Simons invariants. It is also interesting to note that our results maybe well applied to SUSY dilaton coupled theories like Wess-Zumino model (for two-dimensional case, see [38]).

If string theory or other alternative theory (like M-theory) takes over local field theory, the latter formalism should show some theoretical breakdown at some scale such as the Planck mass. At present some pathological phenomena, such as the entropy loss problem in the black hole geometry could look like such a breakdown. We note here that the conformal anomaly is considered to be a key factor in the Hawking radiation phenomenon. In the transitive period from local field theory to string field theory, "intermediate" fields such as the dilaton are thought to play an important role in understanding new concepts involved in the Planck mass physics. The Weyl anomaly occurs quite generally for most ordinary conformally invariant field theories. It shows that the quantization of fields necessarily breaks the scale invariance. It is always related with the ultraviolet regularization. On the other hand it is frequently suggested that string theory could predict some minimal unit of length in space(-time). The effect of such a unit length on its field theory limit could be related to the conformal anomaly phenomenon. We hope the present results are helpful in studying of such problems. 


\section{Appendix A. Anomaly Formula for Scalar Theories on Curved Space}

In this appendix we derive an anomaly formula for the general differential operator

$$
\vec{D}=\sqrt[4]{g}\left(-\nabla^{2} \cdot \mathbf{1}-\mathcal{M}\right) \frac{1}{\sqrt[4]{g}}
$$

which frequently appears in the scalar theories on curved space. $\mathbf{1}$ is the unit matrix with the size of the field indices: $(\mathbf{1})_{i j}=\delta_{i j} ; i, j=1,2, \cdots, N_{s}$. We proceed in three steps. ${ }^{11}$

i) Flat space

For the case of the flat space : $g_{\mu \nu}=\delta_{\mu \nu}$ the operator reduces to $-\partial^{2}-\mathcal{M}$ and the anomaly is known [12, 36] as

$$
\text { Anomaly }=\frac{1}{(4 \pi)^{2}} \operatorname{Tr}\left(\frac{1}{6} \partial^{2} \mathcal{M}+\frac{1}{2} \mathcal{M}^{2}\right)
$$

where "Tr" means "trace" over the field suffixes $i$ : $\operatorname{Tr} \mathcal{M}=\sum_{i=1}^{N_{s}} \mathcal{M}_{i i}$.

ii) General curved space

From the general covariance the most general form which reduces to (66) in the flat space can be expressed as

$$
\begin{array}{r}
\text { Anomaly }=\frac{\sqrt{g}}{(4 \pi)^{2}} \operatorname{Tr}\left\{\frac{1}{6} \nabla^{2} X+\frac{1}{2} X^{2}\right. \\
\left.+\left(a_{1} R_{\mu \nu \lambda \sigma} R^{\mu \nu \lambda \sigma}+a_{2} R_{\mu \nu} R^{\mu \nu}+a_{3} R^{2}+a_{4} \nabla^{2} R\right) \cdot \mathbf{1}\right\} \\
X=\mathcal{M}+c_{1} R
\end{array}
$$

where $a_{1} \cdots a_{4}$ and $c_{1}$ are some constants to be determined.

iii) Determination of constants

First we consider the case $\mathcal{M}=\frac{1}{6} R$ which corresponds to the case: $f(\phi)=$ $1, \xi=-\frac{1}{6}$ in Sec.2. The above result (67) says

$$
N_{s}=1 \quad, \quad \mathcal{M}=\frac{1}{6} R \quad, \quad X=\left(\frac{1}{6}+c_{1}\right) R,
$$

\footnotetext{
11 This approach was taken in the derivation of the counterterm formula 35 .
} 


$$
\begin{array}{r}
\text { Anomaly }=\frac{\sqrt{g}}{(4 \pi)^{2}}\left(\left\{\frac{1}{6}\left(\frac{1}{6}+c_{1}\right)+a_{4}\right\} \nabla^{2} R\right. \\
\left.+a_{1} R_{\mu \nu \lambda \sigma} R^{\mu \nu \lambda \sigma}+a_{2} R_{\mu \nu} R^{\mu \nu}+\left\{\frac{1}{2}\left(\frac{1}{6}+c_{1}\right)^{2}+a_{3}\right\} R^{2}\right)
\end{array}
$$

Comparing this with the well-established result (say, eq.(40) of [12])

$$
\text { Anomaly }=\frac{\sqrt{g}}{(4 \pi)^{2}} \frac{1}{180}\left(-\nabla^{2} R+R_{\mu \nu \lambda \sigma} R^{\mu \nu \lambda \sigma}-R_{\mu \nu} R^{\mu \nu}\right)
$$

we obtain the first set of constraints on the constants.

$$
\begin{array}{r}
a_{1}=\frac{1}{180} \quad, \quad a_{2}=\frac{-1}{180}, \\
\frac{1}{6}\left(\frac{1}{6}+c_{1}\right)+a_{4}=\frac{-1}{180} \quad, \quad \frac{1}{2}\left(\frac{1}{6}+c_{1}\right)^{2}+a_{3}=0 .
\end{array} .
$$

Next we consider the case of the weak gravity $\left(g_{\mu \nu}=\delta_{\mu \nu}+h_{\mu \nu},\left|h_{\mu \nu}\right| \ll 1\right)$ for the general operator (65) in order to fix the value of $c_{1}$. The operator is expanded as

$$
\begin{gathered}
\vec{D}=-\mathbf{1} \partial^{2}-\mathcal{M}+\mathbf{1}\left(h_{\mu \nu} \partial_{\mu} \partial_{\nu}+\partial_{\mu} h_{\mu \lambda} \partial_{\lambda}+\frac{1}{4} \partial^{2} h\right)+O\left(h^{2}\right) \\
\equiv-\mathbf{1} \partial^{2}-W_{\mu \nu} \partial_{\mu} \partial_{\nu}-N_{\lambda} \partial_{\lambda}-M \\
W_{\mu \nu}=-h_{\mu \nu}+O\left(h^{2}\right), N_{\lambda}=-\partial_{\mu} h_{\mu \lambda}+O\left(h^{2}\right), M=\mathcal{M}-\frac{1}{4} \partial^{2} h+O\left(h^{2}\right)
\end{gathered}
$$

Now we focus on the term $\left(c_{1} /(4 \pi)^{2}\right) \sqrt{g} \mathcal{M} R=\left(c_{1} /(4 \pi)^{2}\right) \mathcal{M}\left(\partial^{2} h-\partial_{\mu} \partial_{\nu} h_{\mu \nu}\right)+$ $O\left(h^{2}\right)$ in (67). The conformal anomaly from (171) is independently obtained by the result of [12 ((38), Table I) taking $W_{\mu \nu}, N_{\lambda}, M$ in the reference as given above:

$$
\begin{array}{r}
\frac{1}{(4 \pi)^{2}} \operatorname{Tr}\left(-\frac{1}{12} M \partial^{2} W_{\mu \mu}+\frac{1}{3} M \partial_{\mu} \partial_{\nu} W_{\mu \nu}-\frac{1}{2} M \partial_{\mu} N_{\mu}+\frac{1}{2} M^{2}\right) \\
\sim \frac{-1}{(4 \pi)^{2}} \operatorname{Tr} \mathcal{M}\left(\frac{1}{6} \partial^{2} h-\frac{1}{6} \partial_{\mu} \partial_{\nu} h_{\mu \nu}+O\left(h^{2}\right)\right)=\frac{1}{(4 \pi)^{2}} \sqrt{g}\left(\frac{-1}{6}\right) \operatorname{Tr} \mathcal{M} R .
\end{array}
$$

This shows

$$
c_{1}=-\frac{1}{6}
$$


(70) and (73) fix all constants. Finally we obtain the anomaly formula for (65) as

$$
\begin{array}{r}
\text { Anomaly }=\frac{\sqrt{g}}{(4 \pi)^{2}} \operatorname{Tr}\left\{\frac{1}{6} \nabla^{2} X+\frac{1}{2} X^{2}\right. \\
\left.+\frac{1}{180}\left(R_{\mu \nu \lambda \sigma} R^{\mu \nu \lambda \sigma}-R_{\mu \nu} R^{\mu \nu}+0 \cdot R^{2}-\nabla^{2} R\right) \cdot \mathbf{1}\right\}, \\
X=\mathcal{M}-\frac{1}{6} R \cdot \mathbf{1} .
\end{array}
$$

As noted in the text, this result ( and (41) to be derived in the next appendix ) is the same as the counterterm formula (say of ref. [15, [16, 17]) where dimensional regularization was taken.

\section{Appendix B. Anomaly Formula for Vector Theories on Curved Space}

In this appendix we derive the anomaly formula for the operator

$$
\vec{D}_{\mu}{ }^{\nu}=-g^{1 / 8}\left\{\delta_{\mu}{ }^{\nu} \nabla^{2}+\left(\mathcal{N}^{\lambda}\right)_{\mu}{ }^{\nu} \nabla_{\lambda}+\mathcal{M}_{\mu}{ }^{\nu}\right\} g^{-1 / 8},
$$

which generally appears for the vector fields on the curved background. $\mathcal{N}^{\lambda}$ and $\mathcal{M}$ are general covariants. We proceed in the similar way as in App.A.

i)Flat Space

For the case of flat space $g_{\mu \nu}=\delta_{\mu \nu}$, the formula appears in [12, 36]:

$$
\begin{array}{r}
\vec{D}_{\mu}{ }^{\nu}=-\delta_{\mu}{ }^{\nu} \partial^{2}-\left(\mathcal{N}^{\lambda}\right)_{\mu}{ }_{\mu} \partial_{\lambda}-\mathcal{M}_{\mu}{ }^{\nu}, \\
X_{\mu}{ }^{\nu}=\mathcal{M}_{\mu}{ }^{\nu}-\frac{1}{2}\left(\partial_{\lambda} \mathcal{N}^{\lambda}\right)_{\mu}{ }^{\nu}-\frac{1}{4}\left(\mathcal{N}_{\lambda} \mathcal{N}^{\lambda}\right)_{\mu}{ }^{\nu}, \\
\left(Y^{\mu \nu}\right)_{\alpha}{ }^{\beta}=\frac{1}{2}\left(\partial^{\mu} \mathcal{N}^{\nu}-\partial^{\nu} \mathcal{N}^{\mu}\right)_{\alpha}{ }^{\beta}+\frac{1}{4}\left[\mathcal{N}^{\mu}, \mathcal{N}^{\nu}\right]_{\alpha}{ }^{\beta}, \\
D^{\mu} X_{\alpha}{ }^{\beta}=\partial^{\mu} X_{\alpha}{ }^{\beta}+\frac{1}{2}\left[\mathcal{N}^{\mu}, X\right]_{\alpha}{ }^{\beta}, \\
\text { Anomaly }_{\text {flat }}=\frac{1}{(4 \pi)^{2}} \operatorname{Tr}\left[\frac{1}{6} D^{2} X+\frac{1}{2} X^{2}+\frac{1}{12} Y_{\mu \nu} Y^{\mu \nu}\right],
\end{array}
$$


where the upper and lower indices do not have meaning in present case $\left(g_{\mu \nu}=g^{\mu \nu}=\delta_{\mu}^{\nu}\right.$ are all the Kronecker's delta), but only do have a notational continuity with the next general case.

ii) Curved Space

The anomaly formula can be first expressed as the most general form which reduces to (76) at the flat space limit:

$$
\begin{gathered}
X_{\mu}{ }^{\nu}=\mathcal{M}_{\mu}{ }^{\nu}-\frac{1}{2}\left(\nabla_{\lambda} \mathcal{N}^{\lambda}\right)_{\mu}{ }^{\nu}-\frac{1}{4}\left(\mathcal{N}_{\lambda} \mathcal{N}^{\lambda}\right)_{\mu}{ }^{\nu}+c_{1} \delta_{\mu}^{\nu} R, \\
\left(Y^{\mu \nu}\right)_{\alpha}{ }^{\beta}=\frac{1}{2}\left(\nabla^{\mu} \mathcal{N}^{\nu}-\nabla^{\nu} \mathcal{N}^{\mu}\right)_{\alpha}{ }^{\beta}+\frac{1}{4}\left[\mathcal{N}^{\mu}, \mathcal{N}^{\nu}\right]_{\alpha}{ }^{\beta}+c_{2} R^{\mu \nu}{ }_{\alpha}{ }^{\beta}, \\
D^{\mu} X_{\alpha}{ }^{\beta}=\nabla^{\mu} X_{\alpha}{ }^{\beta}+\frac{1}{2}\left[\mathcal{N}^{\mu}, X\right]_{\alpha}{ }^{\beta}, \\
\text { Anomaly }=\frac{\sqrt{g}}{(4 \pi)^{2}} \operatorname{Tr}\left\{\frac{1}{6} D^{2} X+\frac{1}{2} X^{2}+\frac{1}{12} Y_{\mu \nu} Y^{\mu \nu}\right. \\
\left.+\left(a_{1} R_{\mu \nu \lambda \sigma} R^{\mu \nu \lambda \sigma}+a_{2} R_{\mu \nu} R^{\mu \nu}+a_{3} R^{2}+a_{4} \nabla^{2} R\right) \cdot \mathbf{1}\right\}
\end{gathered}
$$

where $c_{1}, c_{2}, a_{1-4}$ are some constants to be determined.

iii) Determination of constants

iiia) First we consider a special case of $\left(\mathcal{N}^{\lambda}\right)_{\mu}{ }^{\nu}=0, \mathcal{M}_{\mu}{ }^{\nu}=R_{\mu}{ }^{\nu}$, which is a special case of $f(\phi)=1$ in (29) and is the ordinary photon-gravity theory. Comparing the result from (77)

$$
\begin{array}{r}
X_{\mu}{ }^{\nu}=R_{\mu}{ }^{\nu}+c_{1} \delta_{\mu}^{\nu} R \quad, \quad\left(Y^{\mu \nu}\right)_{\alpha}{ }^{\beta}=c_{2} R_{\alpha}^{\mu \nu} \beta \\
\text { Anomaly }=\frac{\sqrt{g}}{(4 \pi)^{2}}\left\{\frac{1}{6} \nabla^{2}\left(R+4 c_{1} R\right)+\frac{1}{2}\left(R_{\mu}{ }^{\nu}+c_{1} \delta_{\mu}^{\nu} R\right)^{2}\right. \\
\left.+\frac{\left(c_{2}\right)^{2}}{12} R_{\alpha}^{\mu \nu}{ }_{\alpha} R_{\mu \nu \beta}^{\alpha}+4 a_{1} R_{\mu \nu \lambda \sigma} R^{\mu \nu \lambda \sigma}+4 a_{2} R_{\mu \nu} R^{\mu \nu}+4 a_{3} R^{2}+4 a_{4} \nabla^{2} R\right\}
\end{array}
$$

with the well-established result (say, eq.(2.14) of [33])

Anomaly $=\frac{\sqrt{g}}{(4 \pi)^{2}}\left\{-\frac{11}{180} R_{\mu \nu \lambda \sigma} R^{\mu \nu \lambda \sigma}+\frac{43}{90} R_{\mu \nu} R^{\mu \nu}-\frac{1}{9} R^{2}+\frac{1}{30} \nabla^{2} R\right\}$,

we obtain

$$
\begin{array}{r}
-\frac{1}{12}\left(c_{2}\right)^{2}+4 a_{1}=-\frac{11}{180} \quad, \quad \frac{1}{2}+4 a_{2}=\frac{43}{90} \\
\frac{1}{2}\left(2 c_{1}+4 c_{1}^{2}\right)+4 a_{3}=-\frac{1}{9} \quad, \quad \frac{1+4 c_{1}}{6}+4 a_{4}=\frac{1}{30}
\end{array} .
$$


We give here a list of the weak-field expansion of the operator (75) for the use in iiib) and iiic).

$$
\begin{array}{r}
g_{\mu \nu}=\delta_{\mu \nu}+h_{\mu \nu}, \quad\left|h_{\mu \nu}\right| \ll 1 \\
\vec{D}_{\alpha}{ }^{\beta}=-\delta_{\alpha}^{\beta} \partial^{2}-\left(W_{\mu \nu}\right)_{\alpha}{ }_{\alpha} \partial_{\mu} \partial_{\nu}-\left(N_{\mu}\right)_{\alpha}{ }^{\beta} \partial_{\mu}-M_{\alpha}{ }^{\beta},\left(W_{\mu \nu}\right)_{\alpha}{ }^{\beta}=-\delta_{\alpha}{ }^{\beta} h_{\mu \nu}+O\left(h^{2}\right) \\
\left(N_{\mu}\right)_{\alpha}^{\beta}=\left(\mathcal{N}^{\mu}\right)_{\alpha}^{\beta}-\delta_{\alpha}^{\beta}\left(\partial_{\lambda} h_{\lambda \mu}-\frac{1}{4} \partial_{\mu} h\right)-\left(\partial_{\mu} h_{\alpha \beta}+\partial_{\alpha} h_{\mu \beta}-\partial_{\beta} h_{\mu \alpha}\right)+O\left(h^{2}\right) \\
M_{\alpha}{ }^{\beta}=\mathcal{M}_{\alpha}^{\beta}-\frac{1}{8} \delta_{\alpha}^{\beta} \partial^{2} h-\frac{1}{2} \partial_{\mu}\left(\partial_{\mu} h_{\alpha \beta}+\partial_{\alpha} h_{\mu \beta}-\partial_{\beta} h_{\mu \alpha}\right) \\
-\frac{1}{8}\left(\mathcal{N}^{\lambda}\right)_{\alpha}{ }^{\beta} \partial_{\lambda} h-\frac{1}{2}\left(\mathcal{N}^{\lambda}\right)_{\alpha}{ }^{\tau}\left(\partial_{\lambda} h_{\beta \tau}+\partial_{\tau} h_{\lambda \beta}-\partial_{\beta} h_{\lambda \tau}\right)+O\left(h^{2}\right) .
\end{array}
$$

iiib) Second we consider the case $\left(\mathcal{N}^{\lambda}\right)_{\mu}{ }^{\nu}=0$ with a general $\mathcal{M}_{\mu}{ }^{\nu}: \vec{D}_{\mu}{ }^{\nu}=$ $-g^{1 / 8}\left\{\delta_{\mu}{ }^{\nu} \nabla^{2}+\mathcal{M}_{\mu}{ }^{\nu}\right\} g^{-1 / 8}$. We focus on the $R \operatorname{Tr} \mathcal{M}$ term. The formula $([7)$ gives

$$
\frac{c_{1}}{(4 \pi)^{2}} R \operatorname{Tr} \mathcal{M}
$$

On the other hand, the anomaly can be independently obtained by the weakfield expansion (81) and the anomaly formula eq.(38) and TABLE I of ref. [12]:

$$
\begin{array}{r}
\frac{1}{(4 \pi)^{2}} \operatorname{Tr}\left(-\frac{1}{12} M \cdot \partial^{2} W_{\mu \mu}+\frac{1}{3} M \cdot \partial_{\mu} \partial_{\nu} W_{\mu \nu}-\frac{1}{2} M \cdot \partial_{\mu} N_{\mu}+\frac{1}{2} M^{2}\right) \\
\sim \frac{1}{(4 \pi)^{2}} \times \frac{1}{6}\left(-\partial^{2} h+\partial_{\mu} \partial_{\nu} h_{\mu \nu}\right) \times \operatorname{Tr} \mathcal{M}=\frac{1}{(4 \pi)^{2}} \times \frac{-1}{6}\left(R+O\left(h^{2}\right)\right) \operatorname{Tr} \mathcal{M},
\end{array}
$$

and we obtain

$$
c_{1}=-\frac{1}{6}
$$

iiic) With the purpose of determining $c_{2}$, we consider the $\mathcal{M}=0$ case with a general $\mathcal{N}^{\lambda}: \vec{D}_{\alpha}{ }^{\beta}=-g^{1 / 8}\left\{\delta_{\alpha}{ }^{\beta} \nabla^{2}+\left(\mathcal{N}^{\lambda}\right)_{\alpha}{ }^{\beta} \nabla_{\lambda}\right\} g^{-1 / 8}$. We focus on the $\left(\mathcal{N}_{\mu} \mathcal{N}_{\nu}\right)_{\alpha \beta} R^{\mu \nu \beta \alpha}$ term in the anomaly. From (77) we have

$$
+\frac{c_{2}}{12}\left(\mathcal{N}_{\mu} \mathcal{N}_{\nu}\right)_{\alpha \beta} R^{\mu \nu \beta \alpha}
$$


We can derive the above result independently in the weak gravity expansion (81). Eq.(43) of [36] gives, as the corresponding $\mathcal{N} \mathcal{N} \partial \partial h$ part,

$$
\begin{gathered}
\frac{1}{(4 \pi)^{2}} \frac{1}{12} \operatorname{Tr} Y_{\mu \nu} Y_{\mu \nu} \sim \frac{1}{(4 \pi)^{2}} \times\left(-\frac{1}{12}\right) \times \frac{1}{2}\left(\partial_{\mu} \partial_{\alpha} h_{\nu \beta}-\partial_{\mu} \partial_{\beta} h_{\nu \alpha}-\mu \leftrightarrow \nu\right)\left(\mathcal{N}^{\mu} \mathcal{N}^{\nu}\right)_{\beta}{ }^{\alpha} \\
\sim \frac{1}{(4 \pi)^{2}} \times\left(+\frac{1}{12}\right) R_{\mu \nu \alpha}\left(\mathcal{N}^{\mu} \mathcal{N}^{\nu}\right)_{\beta}{ }^{\alpha}
\end{gathered}
$$

where $Y_{\mu \nu} \equiv \partial_{\mu} A_{\nu}-\partial_{\nu} A_{\mu}+\left[A_{\mu}, A_{\nu}\right], A_{\mu} \equiv \frac{1}{2}\left(\delta_{\mu \nu}+Z_{\mu \nu}\right) N_{\nu}$ and $\left(\delta_{\mu \nu}+\right.$ $\left.W_{\mu \nu}\right)\left(\delta_{\nu \lambda}+Z_{\nu \lambda}\right)=\delta_{\mu \lambda} \cdot \mathbf{1}$. Therefore we obtain

$$
c_{2}=+1
$$

Finally we obtain

$$
a_{1}=+\frac{1}{180}, a_{2}=-\frac{1}{180}, a_{3}=0, a_{4}=-\frac{1}{180}, c_{1}=-\frac{1}{6}, c_{2}=+1,
$$

and the general formula is given by (41).

\section{Appendix C. Weyl Transformation}

We list here some formulae of Weyl transformation for various fields. Only in this appendix the space(-time) is n-dimensional.

i) Definition

$$
\begin{gathered}
R_{\mu \nu \sigma}^{\lambda}=\partial_{\nu} \Gamma_{\mu \sigma}^{\lambda}+\Gamma_{\tau \nu}^{\lambda} \Gamma_{\mu \sigma}^{\tau}-\nu \leftrightarrow \sigma, \Gamma_{\mu \nu}^{\lambda}=\frac{1}{2} g^{\lambda \sigma}\left(\partial_{\mu} g_{\sigma \nu}+\partial_{\nu} g_{\sigma \mu}-\partial_{\sigma} g_{\mu \nu}\right) \\
R_{\mu \nu}=R_{\mu \nu \lambda}^{\lambda}, R=g^{\mu \nu} R_{\mu \nu}, g=+\operatorname{det} g_{\mu \nu}, \nabla_{\mu} A_{\nu}=\partial_{\mu} A_{\nu}-\Gamma_{\mu \nu}^{\lambda} A_{\lambda} .(89)
\end{gathered}
$$

ii) Weyl transformation of gravitational fields

$g_{\mu \nu}^{\prime}=\mathrm{e}^{2 \alpha(x)} g_{\mu \nu} \quad, \quad \sqrt{g^{\prime}}=\mathrm{e}^{n \alpha} \sqrt{g} \quad, \quad \Gamma_{\mu \nu}^{\lambda}{ }^{\prime}-\Gamma_{\mu \nu}^{\lambda}=\delta_{\nu}^{\lambda} \alpha_{, \mu}+\delta_{\mu}^{\lambda} \alpha_{, \nu}-g_{\mu \nu} \alpha^{, \lambda}$ 
where $\alpha_{, \mu}=\nabla_{\mu} \alpha\left(=\partial_{\mu} \alpha\right), \alpha^{, \lambda}=g^{\lambda \sigma} \alpha_{, \sigma}$. This is the finite transformation, not the infinitesimal one:

$$
\begin{array}{r}
R_{\mu \nu \sigma}^{\lambda}{ }^{\prime}-R_{\mu \nu \sigma}^{\lambda}=\delta_{\sigma}^{\lambda}\left(\alpha_{, \mu \nu}-\alpha_{, \mu} \alpha_{, \nu}+g_{\mu \nu} \alpha_{, \tau} \alpha^{, \tau}\right)+g_{\mu \nu}\left(\alpha_{, \sigma}^{, \lambda}-\alpha^{, \lambda} \alpha_{, \sigma}\right)-(\nu \leftrightarrow \sigma), \\
R_{\mu \nu}{ }^{\prime}-R_{\mu \nu}=g_{\mu \nu} \nabla^{2} \alpha+(n-2)\left(\alpha_{, \mu \nu}-\alpha_{, \mu} \alpha_{, \nu}+g_{\mu \nu} \alpha_{, \lambda} \alpha^{, \lambda}\right), \\
R^{\prime}=\mathrm{e}^{-2 \alpha}\left\{R+2(n-1) \nabla^{2} \alpha+(n-1)(n-2) \alpha_{, \mu} \alpha^{, \mu}\right\},
\end{array}
$$

where $\alpha_{, \mu \nu}=\nabla_{\nu} \nabla_{\mu} \alpha$.

iii) Weyl tensor

The Weyl tensors defined below are invariant under the Weyl transformation:

$$
\begin{aligned}
& C_{\mu \nu \sigma}^{\lambda}=R_{\mu \nu \sigma}^{\lambda}+\frac{1}{n-2}\left(\delta_{\nu}^{\lambda} R_{\mu \sigma}+g_{\mu \sigma} R_{\nu}^{\lambda}-\nu \leftrightarrow \sigma\right) \\
& +\frac{1}{(n-2)(n-1)}\left(\delta_{\sigma}^{\lambda} g_{\mu \nu}-\nu \leftrightarrow \sigma\right) R \quad, \\
& C_{\mu \nu \sigma}^{\lambda}{ }^{\prime}=C_{\mu \nu \sigma}^{\lambda} \quad, \quad C_{\lambda \mu \nu \sigma}=g_{\lambda \tau} C_{\mu \nu \sigma}^{\tau} \quad, \quad C_{\lambda \mu \nu \sigma}{ }^{\prime}=\mathrm{e}^{2 \alpha} C_{\lambda \mu \nu \sigma} .
\end{aligned}
$$

iv) Scalar

$$
\begin{aligned}
& \varphi^{\prime}=\mathrm{e}^{-\frac{n-2}{2} \alpha(x)} \varphi \quad, \quad\left(\nabla_{\mu} \varphi\right)^{\prime}=\mathrm{e}^{-\frac{n-2}{2} \alpha(x)}\left(\nabla_{\mu} \varphi-\frac{n-2}{2} \alpha_{, \mu} \varphi\right) \\
& \left(\nabla^{2} \varphi\right)^{\prime}=\mathrm{e}^{-\frac{n+2}{2} \alpha(x)}\left\{\nabla^{2} \varphi-\frac{n-2}{2} \varphi \nabla^{2} \alpha-\frac{(n-2)^{2}}{4} \alpha_{, \mu} \alpha^{, \mu} \varphi\right\}
\end{aligned}
$$

The well-known conformal coupling is recognized from the relation:

$$
\left[\sqrt{g}\left(\varphi \nabla^{2} \varphi+\frac{n-2}{4(n-1)} R \varphi^{2}\right)\right]^{\prime}=\sqrt{g}\left(\varphi \nabla^{2} \varphi+\frac{n-2}{4(n-1)} R \varphi^{2}\right)
$$

v) Dilaton

$$
\phi^{\prime}=\phi,\left(\nabla_{\mu} \phi\right)^{\prime}=\nabla_{\mu} \phi\left(=\partial_{\mu} \phi\right),\left(\nabla^{2} \phi\right)^{\prime}=\mathrm{e}^{-2 \alpha}\left\{\nabla^{2} \phi+(n-2) \alpha^{, \lambda} \phi_{, \lambda}\right\} .
$$

We note the dilaton and the scalar transform in the same way only in the 2D space:

$$
\begin{array}{r}
\left(\nabla_{\mu} \phi \cdot \nabla^{\mu} \phi\right)^{\prime}=\mathrm{e}^{-2 \alpha} \nabla_{\mu} \phi \cdot \nabla^{\mu} \phi \\
\left(\nabla_{\mu} \nabla_{\nu} \phi\right)^{\prime}=\nabla_{\mu} \nabla_{\nu} \phi-\left(\alpha_{, \mu} \phi_{, \nu}+\alpha_{, \nu} \phi_{, \mu}-g_{\mu \nu} \alpha^{, \lambda} \phi_{, \lambda}\right)
\end{array}
$$


Here we see $\sqrt{g}\left(\nabla_{\mu} \phi \cdot \nabla^{\mu} \phi\right)^{n / 2}$ is conformal invariant in n-dim space. In the text the general form of the dilaton coupling $\mathrm{e}^{P(\phi)}$ is used instead of $\mathrm{e}^{\text {const } \times \phi}$. We may substitute $P(\phi)$ for $\phi$ in the above formulae (e.g. $P_{, \mu \nu}{ }^{\prime}=$ $\left.P_{, \mu \nu}-\left(\alpha_{, \mu} P_{, \nu}+\alpha_{, \nu} P_{, \mu}-g_{\mu \nu} \alpha^{, \lambda} P_{, \lambda}\right)\right)$.

\section{Appendix D. Weyl Invariants and Trivial Terms}

We consider $4 \mathrm{D}$ space in this appendix. In the present model we have the following conformal invariants made only of the metric and the dilaton field:

$$
\begin{gathered}
I_{0} \equiv \sqrt{g} C_{\mu \nu \lambda \sigma} C^{\mu \nu \lambda \sigma} \quad, \quad I_{4} \equiv \sqrt{g}\left(P_{, \mu} P^{, \mu}\right)^{2} \\
I_{2} \equiv \sqrt{g}\left(R^{\mu \nu} P_{, \mu} P_{, \nu}-\frac{1}{6} R P_{, \mu} P^{, \mu}+\frac{3}{4}\left(\nabla^{2} P\right)^{2}-\frac{1}{2} P^{, \mu \nu} P_{, \mu \nu}\right) \\
I_{1} \equiv \sqrt{g}\left(R^{\mu \nu}-\frac{1}{2} g^{\mu \nu} R\right) P_{, \mu \nu}=\sqrt{g} \nabla_{\nu}\left\{\left(R^{\mu \nu}-\frac{1}{2} g^{\mu \nu} R\right) P_{, \mu}\right\}
\end{gathered},
$$

where the numbers in the lower suffixes show the numbers of powers $P$. They transform under the finite Weyl transformation as

$$
\begin{gathered}
I_{0}{ }^{\prime}=I_{0} \quad, \quad I_{4}{ }^{\prime}=I_{4} \\
I_{2}{ }^{\prime}-I_{2}=2 \sqrt{g} \nabla^{\mu}\left(\alpha^{, \nu} P_{, \mu} P_{, \nu}\right) \\
I_{1}{ }^{\prime}-I_{1}=\sqrt{g} \nabla^{\nu}\left\{2\left(P_{, \mu \nu} \alpha^{, \mu}-\nabla^{2} P \cdot \nabla_{\nu} \alpha\right)-\left(2 \alpha^{, \mu} \alpha_{, \nu} P_{, \mu}+\alpha_{, \mu} \alpha^{, \mu} P_{, \nu}\right)\right\}
\end{gathered}
$$

Two terms $I_{2}$ and $I_{1}$, which are conformally invariant up to total derivative terms, may be called generalized conformal invariants. They lead to strange consequences, in comparison with standard conformal invariants, in construction of the anomaly induced effective action. As the quantities with the same properties, we note the Euler term, $\sqrt{g} E$, defined in (26) and a trivial term, $J_{0}$, defined below.

$$
\begin{gathered}
(\sqrt{g} E)^{\prime}-\sqrt{g} E=4 \sqrt{g} \nabla^{\mu}\left\{R \alpha_{, \mu}-2 R_{\mu}^{\nu} \alpha_{, \nu}-\nabla_{\mu}\left(\alpha_{, \nu} \alpha^{, \nu}\right)+2 \alpha_{, \mu} \nabla^{2} \alpha+2 \alpha_{, \nu} \alpha^{, \nu} \alpha_{, \mu}\right\} \\
\frac{2}{3}\left(J_{0}{ }^{\prime}-J_{0}\right)=4 \sqrt{g} \nabla^{\mu}\left\{\nabla_{\mu}\left(\nabla^{2} \alpha+\alpha_{, \nu} \alpha^{, \nu}\right)-\alpha_{, \mu}\left(\frac{1}{3} R+2 \nabla^{2} \alpha+2 \alpha_{, \nu} \alpha^{, \nu}\right)\right\} \\
\left(\sqrt{g} E+\frac{2}{3} J_{0}\right)^{\prime}-\left(\sqrt{g} E+\frac{2}{3} J_{0}\right)=4 \sqrt{g} \Delta \alpha
\end{gathered}
$$

where $\sqrt{g} \Delta$ is the conformally invariant 4 -th order differential operator defined in eq. (62) of the text. 
Among the Weyl anomaly terms there are those terms which can be obtained by the infinitesimal Weyl transformation of local (counter)terms composed only of the background fields $\left(g_{\mu \nu}, \phi\right)$. They are called "trivial" (anomaly) terms [21]. ( In the mathematical (cohomology) terminology, it is called coboundary [37]). To obtain them, it is sufficient to consider the infinitesimal transformation $(\alpha(x) \ll 1)$ in the appendix $\mathrm{C}$ :

$$
\begin{gathered}
J_{0} \equiv \sqrt{g} \nabla^{2} R, K_{0} \equiv \sqrt{g} R^{2}, g_{\mu \nu} \frac{\delta}{\delta g_{\mu \nu}(x)} \int d^{4} x K_{0}=6 J_{0}, \\
J_{2 a} \equiv \sqrt{g} \nabla^{2}\left(P_{, \mu} P^{, \mu}\right), K_{2 a} \equiv \sqrt{g} R P_{, \mu} P^{, \mu}, g_{\mu \nu} \frac{\delta}{\delta g_{\mu \nu}(x)} \int d^{4} x K_{2 a}=+3 J_{2 a}, \\
J_{2 b} \equiv \sqrt{g}\left(-R^{\mu \nu} P_{, \mu} P_{, \nu}+P_{, \mu \nu} P^{, \mu \nu}-\left(\nabla^{2} P\right)^{2}\right)=\sqrt{g} \nabla_{\mu}\left(P^{, \mu \nu} P_{, \nu}-P^{, \mu} \nabla^{2} P\right), \\
K_{2 b} \equiv \sqrt{g}\left(P^{, \mu \nu} P_{, \mu \nu}+\frac{1}{2}\left(\nabla^{2} P\right)^{2}\right), g_{\mu \nu} \frac{\delta}{\delta g_{\mu \nu}(x)} \int d^{4} x K_{2 b}=+2 J_{2 b}, \\
J_{2 c} \equiv \sqrt{g} \nabla_{\mu} \nabla_{\nu}\left(P^{, \mu} P^{, \nu}\right), K_{2 c} \equiv \sqrt{g}\left(R^{\mu \nu} P_{, \mu} P_{, \nu}-\frac{1}{6} R P_{, \mu} P^{, \mu}\right), \\
g_{\mu \nu} \frac{\delta}{\delta g_{\mu \nu}(x)} \int d^{4} x K_{2 c}=+J_{2 c}, \\
J_{2 d} \equiv \sqrt{g} \nabla_{\mu}\left(P^{, \mu \nu} P_{, \nu}\right), K_{2 d} \equiv \sqrt{g}\left(P_{, \mu \nu} P^{, \mu \nu}-\frac{1}{2}\left(\nabla^{2} P\right)^{2}\right), \\
g_{\mu \nu} \frac{\delta}{\delta g_{\mu \nu}(x)} \int d^{4} x K_{2 d}=+2 J_{2 d}, \\
J_{3} \equiv \sqrt{g}\left(\nabla^{2} P \cdot P_{, \mu} P^{, \mu}+2 P^{, \mu} P^{, \nu} P_{, \mu \nu}\right)=\sqrt{g} \nabla^{\nu}\left(P_{, \nu} P_{, \mu} P^{, \mu}\right), \\
K_{3} \equiv \sqrt{g} P^{, \mu \nu} P_{, \mu} P_{, \nu}, g_{\mu \nu} \frac{\delta}{\delta g_{\mu \nu}(x)} \int d^{4} x K_{3}=\frac{1}{2} J_{3}, \\
J_{1} \equiv \sqrt{g}\left(3 \nabla^{4} P-\nabla^{\lambda}\left(R P_{, \lambda}\right)\right), K_{1} \equiv \sqrt{g} R \nabla^{2} P, g_{\mu \nu} \frac{\delta}{\delta g_{\mu \nu}(x)} \int d^{4} x K_{1}=J(100)
\end{gathered}
$$

$J$ 's are trivial terms, while $K$ 's are the corresponding counterterms. We note all trivial terms are total derivative ones. But total derivative terms are not always trivial ones. $I_{1}$ is such an example $\square$.

\section{Appendix E. Conformal Anomaly for 2D Dilaton Coupled Scalar}

For completeness we write below the conformal anomaly for 2D dilaton

\footnotetext{
${ }^{12} I_{1}$ looks like the Euler term in the sense that its Weyl transformation is similar, as pointed out below (98), and it is the surface term which depends only on the topology.
} 
coupled scalar with the action:

$$
S=\frac{1}{2} \int d^{2} x \sqrt{g} \mathrm{e}^{-2 \phi}(\nabla \varphi)^{2}
$$

The notation is the same as in Sec.2 of the text. The calculation of the corresponding trace anomaly has been done actually in Ref. [3] (see later

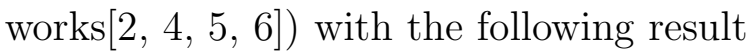

$$
T=\frac{\sqrt{g}}{24 \pi}\left\{-R-6(\nabla \phi)^{2}+6 \nabla^{2} \phi\right\} .
$$

The structure of the above anomaly consists of the Euler term, $\sqrt{g} R$, a trivial term, $\sqrt{g} \nabla^{2} \phi$, and a conformally invariant term, $\sqrt{g}(\nabla \phi)^{2}$. So the structure is the same as $4 \mathrm{D}$ dilaton coupled scalar in Sec.2 of the text.

The analysis given in above works leads to different values for the coefficient of the trivial term. In particulary, the result of ref. [2] does not coincide with the calculations of refs. [3, 4, 5, 6]. The discrepancy occurs because of the difference in the choice of the ultraviolet regularization, the system operator and the measure[5]. The most crucial point is, as far as we are studying the anomaly problem in the perturbative quantization of the matter fields only, we have no control over the purely gravity-dilaton sector. It means the corresponding coefficient appears to be ambigious. We have the freedom to introduce a finite counterterm $\sqrt{g} R \phi$ in the local gravity-dilaton action. (As for the the most general form of such a local action, see Ref.[39].) This changes the coefficient of the trivial term freely due to relation:

$$
g_{\mu \nu} \frac{\delta}{\delta g_{\mu \nu}(x)} \int d^{2} x \sqrt{g} R \phi=\sqrt{g} \nabla^{2} \phi
$$

Hence, the coefficient of the trivial term is ambigious (actually, it is defined by the normalization condition). Looking at the situation in the 2D black hole physics, the radiation amplitude is determined by the conformal anomaly equation and conservation of the energy- momentum tensor. The trivial term equally contributes to its determination. If we regard the radiation amplitude to be physical, it appears that we have to treat the the matter-gravity-dilaton system both nonperturbatively and on an equal footing with all fields. 


\section{Acknowledgements}

The authors thank K.Fujikawa and R.Kantowski for reading the manuscript and some remarks. SI thanks R.Endo for some comments. SDO is grateful to I.Buchbinder and S.Nojiri for related discussions.

\section{References}

[1] S.K.Rama,Phys.Rev.Lett.78,1620(1997).

[2] R.Bousso and S.W.Hawking, Phys.Rev.D56,7788(1997), hep-th/9705236.

[3] E.Elizalde,S.Naftulin and S.D.Odintsov,Phys.Rev.D49,2852(1994).

[4] S.Nojiri and S.D.Odintsov, Mod.Phys.Lett.A12,2083(1997), hepth/9706009.

[5] S.Ichinose, Phys.Rev.D57,6224(1998), hep-th/9707025.

[6] W.Kummer,H.Liebl

and

D.V.Vassilevich,Mod.Phys.Lett.A12,2683(1997), hep-th/9707041.

[7] J.Erdmenger and H.Osborn,Nucl.Phys.B483,431(1997).

[8] J.L.Cardy, Nucl.Phys.B366,403(1991).

[9] S.Nojiri and S.D.Odintsov,Phys.Lett.B426,29(1998), hep-th/9801052.

[10] S.Ichinose,US-97-08,hep-th/9801056, "General Structure of Conformal Anomaly and 4 Dimensional Photon-Dilaton Gravity"

[11] K.Fujikawa,Phys.Rev.Lett.42,1195(1979);441733(1980);

Phys.Rev.D21,2848(1980); D22,1499(E)(1980).

[12] S.Ichinose and N.Ikeda,Phys.Rev.D53,5932(1996).

[13] L.Bonora and P.Cotta-Ramusino,Phys.Lett.126B,305(1983). 
[14] K.Fujikawa,Nucl.Phys.B226,437(1983).

[15] A.O.Barvinsky and G.A.Vilkovisky,Nucl.Phys.B191,237(1981).

[16] A.O.Barvinsky and G.A.Vilkovisky,Phys.Rep.C119,1(1985).

[17] I.L.Buchbinder,S.D.Odintsov and I.L.Shapiro,Effective Action in Quantum Gravity, IOP Publication, Bristol and Philadelphia, 1992.

[18] S.Nojiri and S.D.Odintsov,Phys.Rev.D57,2363(1998), hep-th/9706143.

[19] S.Deser, M.J.Duff, and C.J.Isham,Nucl.Phys.B111,45(1976)

[20] M.J.Duff,Nucl.Phys.B125,334(1977).

[21] S.Deser and A.Schwimmer,Phys.Lett.B309,279(1993).

[22] K.Fujikawa,Phys.Rev.D23,2262(1981).

[23] L.S.Brown and J.P.Cassidy,Phys.Rev.D15,2810(1977);Phys.Rev.D16,1712(1977).

[24] J.S.Dowker and R.Critchley,Phys.Rev.D16,3390(1977).

[25] S.Christensen and M.Duff,Nucl.Phys.B154,301(1979).

[26] K.Fujikawa,Lecture in Quantum Gravity and Cosmology, Kyoto Summer Inst.1985,ed.by Sato and Inami,World Scientific :106 (1985) .

[27] J.Wess and J.Bagger,"Supersymmetry and Supergravity", 2nd edition, Princeton Univ.Press,Princeton,New Jersey,C1992.

[28] R.Riegert,Phys.Lett.B134,56(1984).

[29] E.S.Fradkin and A.Tseytlin,Phys.Lett.B134,187(1984).

[30] L.B.Buchbinder, S.D.Odintsov and I.L.Shapiro, Phys.Lett.B162,92(1985).

[31] I.Antoniadis and E.Mottola,Phys.Rev.D45,2013(1992).

[32] S.D.Odintsov,Z.Phys.C54,531(1992). 
[33] R.Endo,Prog.Theor.Phys.71,1366(1984).

[34] N.K.Nielsen and P.van Nieuwenhuizen,Phys.Rev.D38,3183(1988).

[35] S.Ichinose and M.Omote,Nucl.Phys.B203,221(1982).

[36] S.Ichinose and N.Ikeda,DAMTP/96-87, hep-th/9610136, "Gauge Symmetry of the Heat Kernel and Anomaly Formulae".

[37] L.Bonora,P.Pasti and M.Bregola,Class.Quantum.Grav.3,635(1986).

[38] S.Nojiri and S.D.Odintsov,Phys.Lett.B416,85(1998), hep-th 9708139.

[39] S.Odintsov and I.Shapiro,Phys.Lett.B263,183(1991). 